\title{
„Man muss sich darauf einlassen, auf die dänische Mentalität“ - Funktionale Angemessenheit in der deutsch-dänischen interkultu- rellen Kommunikation
}

\author{
Katarina Le Müller und Erla Hallsteinsdóttir (Odense)
}

\begin{abstract}
This paper is based on results of the research project National stereotypes and marketing strategies in the intercultural Danish-German communication (SMiK). In the SMiK-project, we define stereotypes as linguistically and culturally determined patterns of thinking and behaving. Such patterns affect intercultural interactions and they should therefore be taken into consideration in the teaching of foreign languages and in intercultural communication. In the SMiK-project we have developed guidelines for the adequate intercultural interaction in the German-Danish business communication. Those guidelines are based on empirical studies on German-Danish national stereotypes. In this paper, we will discuss the theoretical and methodological approach to the compilation of these guidelines and their background in studies on intercultural communication and in the Sprachkritik-paradigm (a linguistically founded critical-reflecting appraisal of language use) will be described. We hereby focus on the concepts of 'Weltansichten' as conceptualizations of the world, cultural awareness and functional adequacy in the intercultural communication. Finally, we give examples for the implementation of the results from an analyses of interview data in guidelines for Danish-German business communication.
\end{abstract}

\section{$1 \quad$ Einleitung}

Ein wichtiges Ziel des SMiK-Projekts ${ }^{1}$ ist die empirische Erarbeitung von neuem Wissen über aktuelle deutsch-dänische Stereotype, d. h. die Auffassungen, die Menschen in Deutschland und Dänemark voneinander haben. Auf der Grundlage des im Projekt gewonnenen Wissens werden wissenschaftlich abgesicherte Unterrichtsmaterialien und Hilfsmittel für die Bewusstmachung von nationalen Stereotypen im Unterricht Deutsch und Dänisch als Fremdsprache (cf. Baunsgaard Koll/Heinz in diesem Heft; Hallsteinsdóttir et al. 2015) sowie Ratgeber für die interkulturelle Kommunikation erstellt.

\footnotetext{
${ }^{1}$ Das Projekt Nationale Stereotype und Marketingstrategien in der interkulturellen deutsch-dänischen Kommunikation (SMiK; www.stereotypenprojekt.eu [13.02.2016]) wurde gefördert durch INTERREG4A SyddanmarkSchleswig-K.E.R.N. mit Mitteln des Europäischen Fonds für regionale Entwicklung; www.interreg4a.de/wm390752 [13.02.2016].
}

Linguistik online 79, 5/2016 - http://dx.doi.org/10.13092/lo.79.3346

CC by 3.0 
In diesem Beitrag beschreiben wir die wissenschaftliche Grundlage und Erarbeitung von Ratgebermaterialien für die interkulturelle Kommunikation zwischen Menschen in Deutschland und Dänemark. Diese Materialien enthalten exemplarische Beispiele (best practice) mit Fokus auf die deutsch-dänische Kommunikation in wirtschaftlichen Kontexten. Die empirische Grundlage für die Materialien bilden Interviews mit kleinen und mittelständischen Firmen auf beiden Seiten der deutsch-dänischen Grenze.

Wir werden in diesem Beitrag den Weg nachzeichnen, den wir von der Erhebung von Interviewdaten zur Fertigstellung von Ratgebern ${ }^{2}$ für die deutsch-dänische Kommunikation in der Praxis beschritten haben. Zunächst diskutieren wir das gewählte theoretische Konzept des Anderen aus der Forschung zur interkulturellen Kommunikation und das Konzept der funktionalen Angemessenheit aus der linguistischen Sprachkritik, die die theoretisch-methodischen Rahmenbedingungen für die auszuarbeitenden Materialien für die Praxis bilden. Schließlich zeigen wir, wie wir auf der Grundlage von Interviewdaten aus einer Interviewbefragung mit 41 Unternehmen und anderen Akteuren aus dem deutsch-dänischen Grenzgebiet das Wissen über die erlebten und vermuteten Unterschiede zwischen Deutschen und Dänen, die von den Interviewten beiderseits der deutsch-dänischen Grenze formuliert wurden, gezielt in Ratgebern für die deutsch-dänische Kommunikation umgesetzt haben.

\section{Theoretischer Hintergrund}

„Eine Gesellschaft hat keine Kultur, sondern ist Kultur.“ (Bolten 2007b: 14)

Die theoretische Grundlage der Ausarbeitung von den Ratgebermaterialien des SMiKProjekts zur interkulturellen deutsch-dänischen Kommunikation bilden insbesondere die Überlegungen von Bolten zur interkulturellen Kommunikation (cf. Bolten 2007a, 2007b, 2012, 2015) und das Konzept der funktionalen Angemessenheit aus der linguistischen Sprachkritik (cf. Kilian 2001, 2003, 2005, 2008; Kilian/Niehr/Schiewe 2010, 2013a, 2013b). Auf eine ausführliche Auseinandersetzung mit den vielfältigen theoretischen Ansätzen der interkulturellen Kommunikation und den verschiedenen Ansätzen innerhalb der linguistischen Sprachkritik wird an dieser Stelle aus Platzgründen verzichtet.

\subsection{Lebenswelten und Weltansichten}

Kultur fassen wir in Anlehnung an Bolten (2007a), ,als konventionalisierte (und damit i. w. S. historisch tradierte) Reziprozitätspraxis“ (Bolten 2007a: 42) auf. Kultur basiert auf kulturellen Wissensvorräten (cf. Bolten 2007a: 75), die in Form von

\footnotetext{
2 Die Daten wurden in Ratgebern zur deutsch-dänischen Kommunikation (cf. Müller/Hallsteinsdóttir 2015a, 2015b, 2015c, 2015d) umgesetzt. Die Ratgeber sind auf der SMiK-Homepage www.stereotypenprojekt.eu [13.02.2016] unter „Projektresultate(r)“ zu finden. Wir beziehen uns insbesondere auf die deutschsprachige Version Deutsch-dänische Kulturbrille (Hallsteinsdóttir/Müller 2015b).
} 
auf die dänische Mentalität"

Wissens-, Bedeutungs- oder Sinnsysteme[n] [...], die soziales Handeln erst ermöglichen, indem sie auf Dauer überindividuelle Wirklichkeitskonstruktionen vorgeben, Orientierungsmuster anbieten und Identität(en) konstruieren.

(Antos/Pogner 2003: 396, zitiert nach Fix 2006: 259)

Kultur im weiten Sinne manifestiert sich als die Lebenswelten von Individuen einer Gesellschaft (cf. Bolten 2007b: 13-14)3. Innerhalb einer Gesellschaft können mehrere Lebenswelten existieren und jeder Mensch kann auch mehreren Lebenswelten angehören, in denen sie oder er unterschiedliche Rollen spielt.

Eine konkrete Fixierung des Kulturbegriffs erfolgt in unserer Arbeit vorerst einerseits sprachlich durch die jeweilige deutsche bzw. dänische Sprachgemeinschaft und andererseits geografisch durch die zu untersuchenden nationalen sprachlich-kulturellen Stereotype (cf. Bolten 2007b: 15). Zur methodischen Abgrenzung der im Projekt durchgeführten Untersuchungen wird demnach zunächst eine geschlossen-räumlich fixierte Definition der untersuchten Kulturen durch die jeweils dazugehörige nationale Sprache und Kultur vorgenommen. Für die theoretische Einordnung der Ergebnisse und die Ausarbeitung der Ratgebermaterialien arbeiten wir jedoch mit einer offenen, sozial fixierten Kulturauffassung, nach der jeder Sprecher zu mehreren (Sub-)Kulturen im sozio-linguistischen Sinne gehört ${ }^{4}$, und somit innerhalb jeder Sprache mehrere Kulturen bzw. (Sub-)Lebenswelten existieren können (cf. Bolten 2007b).

Die individuelle Lebenswelt wird durch Stereotype, d. h. sprachliche und kulturelle Denkund Handlungsmuster und Vorstellungen, strukturiert, die unsere Wahrnehmung prägen. Solche generalisierenden Muster bilden die Grundlage für unsere Weltansichten, die wir in den Ratgebern bildhaft als Kulturbrillen darstellen. Stereotype Muster definieren die Normalitätserwartungen, die unsere alltäglichen und häufig unbewussten sprachlichen und kulturellen Routinehandlungen beeinflussen. Dadurch, dass unsere eigene Lebenswelt die Normalität repräsentiert, sind wir uns der Kulturgebundenheit unserer Handlungen und Wahrnehmungen, d. h. dass wir die Welt durch die Normen, Werte und Erwartungen einer Kulturbrille betrachten, meist nur bedingt bewusst.

\subsection{Stereotype Selbst-, Fremd- und Metabilder}

Dass es einen Unterschied zwischen den Bildern in unseren Köpfen und der wirklichen Welt gibt, darauf hat Walter Lippmann bereits 1922 hingewiesen. Die ,pictures in our heads“ (Lippmann 1922: 1) als die stereotypen Vorstellungen, die wir von uns selbst und von anderen haben, können in Auto-, Hetero- und Spiegelstereotype aufgeteilt werden. Autostereotype sind die Vorstellungen und das Selbstbild, die wir von uns selbst und den Eigengruppen in unseren Lebenswelten haben. Heterostereotype sind die Bilder und Vorstellungen, die wir von anderen Gruppen, Nationen etc. haben, die nicht zur eigenen Lebenswelt gehören. Das

\footnotetext{
${ }^{3}$ Aufgrund der unzähligen Definitionen von Kultur (cf. Bolten 2014) ist eine engere Definition von Kultur ohne eine ausführliche Auseinandersetzung mit der dazugehörigen Literatur wenig sinnvoll. Diese würde den Rahmen dieses Beitrages sprengen.

${ }^{4}$ Umgekehrt gilt auch, dass es Kulturen gibt, in denen sich die Mitglieder mehrerer Sprachen bedienen, wie z. B. in den deutsch-dänischen Minderheiten.
} 
Selbstbild und die Autostereotype der eigenen Lebenswelt bilden die Grundlage dessen, wie wir andere Menschen auffassen und mit ihnen umgehen:

Fest steht, dass ein Selbstverständnis nicht möglich wäre, wenn es nicht den „Anderen“, „Fremden“ gäbe, mit dem ich mich vergleichen könnte. Umgekehrt ist auch mein Verständnis des Fremden in erster Linie davon abhängig, wie ich mich selbst in dieser Beziehung sehe.

(Bolten 2012: 80)

Spiegelstereotype sind die „Metabilder“ (Bolten 2012: 82) und die Vorstellungen, von denen Menschen glauben, dass sie die Mitglieder einer anderen Lebenswelt von ihnen und ihrer Lebenswelt haben:

Unsere Wahrnehmung von Eigenem und Fremden ist über das direkte Wechselspiel von Selbstund Fremdverstehen hinaus auch wesentlich durch das geprägt, was wir annehmen bzw. vermuten, was andere von uns denken und erwarten. Man spricht in diesem Zusammenhang von „Metabildern". Wenn ich z. B. vermute, dass der Andere von mir erwartet, dass ich in einer bestimmten Kleidung zu einer Veranstaltung gehe, die derjenige auch besucht, so kann diese Vermutung bzw. dieses Metabild für mich durchaus handlungsleitend sein und mich zur Wahl entsprechender Kleidungsstücke motivieren (die ich „von mir aus“ eventuell gar nicht in Betracht gezogen hätte). Hieraus folgt: Wenn wir Fremdes (und Eigenes) wahrnehmen und verstehen, dann geschieht dies auf der Grundlage des wechselseitigen Zusammenhangs von Selbst-, Fremd- und Metabildern.

(Bolten 2012: 82)

Unterschiede in der Wahrnehmung von Meta-, Selbst- und Fremdbildern und den mit diesen Bildern verbundenen Denk- und Handlungsmustern können zu Missverständnissen in der interkulturellen Kommunikation führen:

Viele interkulturelle Missverständnisse und Probleme resultieren daraus, dass man sich der Kulturgebundenheit der eigenen und der Wahrnehmung seines fremdkulturellen Partners nicht hinreichend bewusst ist. Es werden Dinge und Sachverhalte unhinterfragt als „normal“ angesehen, die für die Wahrnehmungsgewohnheiten des anderen keineswegs plausibel sind.

(Bolten 2012: 50)

Daher ist eine grundlegende Voraussetzung für das Gelingen der interkulturellen Kommunikation die Reflexion darüber, welche sprachlich-kulturellen Weltansichten, Konventionen, Normalitätsauffassungen und Erwartungshaltungen man als Teil des eigenen Selbstbilds hat. Wenn man die Kulturgebundenheit seiner eigenen Lebenswelt kennt und sich somit der prägenden Perspektive seiner Weltansichten, also seiner Kulturbrille, bewusst ist, dann kann man andere Lebenswelten differenziert betrachten, ihre Kulturgebundenheit verstehen und ihre Normalitätsauffassungen akzeptieren. Die Bewusstmachung von Auto-, Hetero- und Spiegelstereotypen als sprachlich-kulturellen Mustern in den Weltansichten der Menschen gehört daher zu den Grundlagen für die SMiK-Ratgeber. Bei den aktuellen deutsch-dänischen Stereotypen können wir auf Ergebnisse aus anderen Untersuchungen des SMiK-Projekts zurückgreifen. Diese Untersuchungen zeigen, dass sich die deutsch-dänischen Auto-, Hetero- und Spiegelstereotype unterscheiden. Das Fremd- und Metabild, das Deutsche kollektiv-usuell von Dänen haben - und umgekehrt -, stimmt nicht überein mit dem deutschen bzw. dänischen Selbstbild (cf. Hallsteinsdóttir 2015: 12-15; Hallsteinsdóttir/Kilian 2015; Hof- 
auf die dänische Mentalität“"

mann/Hallsteinsdóttir in diesem Heft; Müller 2016; Müller/Hallsteinsdóttir 2016). Die Verwendung des Kollektivplurals impliziert hier keinesfalls, dass alle Deutschen bzw. alle Dänen gleich sind oder sich gleich verhalten. Wir schließen uns vielmehr der Auffassung von Jürgen Bolten an, dass für die interkulturelle Kommunikation ,,jede Verallgemeinerung im Sinne von , die Deutschen sind <..>` strikt unzulässig“" (Bolten 2012: 65) sei.

\subsection{Interkulturelle Kommunikation}

Interkulturelle Kommunikation findet zwischen einzelnen Menschen aus unterschiedlichen Kulturen im Sinne von Lebenswelten und Weltansichten statt. Die interkulturelle Kommunikation ist somit ein individueller Aushandlungsprozess, in dem die Handlungsmuster ausgehandelt werden. Welche Handlungsmuster in einer Situation möglich und angemessen sind, hängt von diversen Variablen ab, die von kulturellen und sprachlichen Konventionen in den Lebenswelten bestimmt und für konkrete Situationen definiert werden können (wie Alter, Bekanntheitsgrad, Hierarchiegefälle, cf. Bolten 2012: 40-41 - cf. auch Abschnitt 2.3 weiter unten).

In der Interaktion zwischen Menschen aus unterschiedlichen Lebenswelten in der interkulturellen Kommunikation entstehen Interkulturen:

Offenkundig ist zunächst, dass eine solche Interkultur nicht abstrakt, d. h. zwischen „den“ Deutschen und „den“ Polen, sondern immer nur vermittelt über konkrete Individuen geschehen kann. Interkulturen entstehen folglich dann, wenn Mitglieder unterschiedlicher Lebenswelten A und B miteinander interagieren, gemeinschaftlich handeln. Umgekehrt existieren Interkulturen auch nur in Abhängigkeit von den daran Beteiligten. Aus diesem Grund „ereignen“ sie sich. Interkulturen werden permanent neu erzeugt, und zwar im Sinne eines „Dritten“ einer Zwischen-Welt $\mathrm{C}$, die weder der Lebenswelt A noch der Lebenswelt B vollkommen entspricht.

(Bolten 2012: 40)

Im Folgenden werden nur kurz einige Begriffe der interkulturellen Kommunikation erläutert, die die theoretische Grundlage der Ratgeber beeinflusst haben. Wir unterscheiden in Anlehnung an Bolten (2007b, 2012) zwischen Multikulturalität und Interkulturalität, und führen den Begriff Mehrkulturalität ein:

- Multikulturalität ist eine Eigenschaft von Lebenswelten, die sich aus Mitgliedern mit unterschiedlichen Kulturen zusammensetzt, d. h. der Begriff bezeichnet die soziale Organisationsstruktur einer multikulturellen Gesellschaft (cf. Bolten 2012: 39-40).

- Interkulturalität umfasst das Interagieren der Menschen als soziale Prozesse im Zusammenleben in einer multikulturellen Lebenswelt (cf. Bolten 2012: 39-40).

- Mehrkulturalität bezeichnet die Synergie von mehreren Kulturen in den Köpfen der Menschen, die interkulturell agieren.

Der Begriff interkulturelle Kommunikation wird häufig so verstanden, dass die Teilnehmenden zwischen unterschiedlichen Kulturen kommunizieren, und dass diese Kulturen voneinander abgegrenzte Einheiten bilden. Wenn man sich jedoch als Individuum mit mehreren Kulturen beschäftigt, z. B. wenn man zwischen mehreren Kulturen kommuniziert, dann trennt 
man diese Kulturen nicht. Interkulturell agierende Sprecher nehmen vielmehr die anderen Kulturen auf und implementieren ihr Wissen darüber in ihrer eigenkulturellen Lebenswelt. Ihre interkulturelle Kompetenz und ihr interkulturelles Verständnis beruhen somit darauf, dass die Sprecher eine innere Mehrkulturalität aufbauen, die mehr und anderes ist als die Summe der darin vorkommenden Einzelkulturen. Sie kommunizieren dann zwar zwischen unterschiedlichen Kulturen, aber sie befinden sich auch gleichzeitig innerhalb von mehreren Kulturen, die zusammenwirken.

\subsection{Interkulturelle Kompetenz}

Die Frage, ob es eine spezielle interkulturelle Kompetenz als eine Schlüsselkompetenz für die interkulturelle Kommunikation gebe, wird unterschiedlich beantwortet. Wir halten uns weiterhin an die Ausführungen von Bolten (2007b, 2012). Er definiert ,interkulturelle Kompetenz als das erfolgreiche ganzheitliche Zusammenspiel von individuellem, sozialem, fachlichem und strategischen Handeln in interkulturellen Kontexten“ (Bolten 2007b: 87).

Diese Aspekte sind grundlegend für erfolgreiches Handeln in allen Kontexten, sowohl eigenals auch interkulturellen. Demnach ist das, was man üblicherweise als interkulturelle Kompetenz bezeichnet, „nur“ die gelungene Verlegung der ohnehin vorhandenen Kompetenzen der Sprecher in einen interkulturellen Kontext, inklusive die Umsetzung im erfolgreichen Handeln:

Interkulturelle Kompetenz stellt keinen eigenständigen Kompetenzbereich dar, sondern ist im Sinne von lat. competere: ,zusammenbringen“ am besten als Fähigkeit zu verstehen, individuelle, soziale, fachliche und strategische Teilkompetenzen in ihrer bestmöglichen Verknüpfung auf interkulturelle Handlungskontexte beziehen zu können. Interkulturelle Kompetenz ist dementsprechend keine Schlüsselqualifikation, sondern eine Querschnittsaufgabe, deren Gelingen das Zusammenspiel verschiedener Schlüsselqualifikationen voraussetzt.

(Bolten 2012: 165)

Die interkulturelle Kompetenz ist somit als eine Fähigkeit einzuordnen, die die individuell vorhandenen eigenkulturellen Schlüsselkompetenzen - also die Aspekte, die für erfolgreiches Handeln in allen, d. h. sowohl eigen- als auch interkulturellen Kontexten, grundlegend sind in einer Interkultur zu verknüpfen. Als besonders wichtige Fähigkeiten gelten (cf. Bolten 2012: 111-115):

- Empathie: sich in die Erfahrungswelten, Normen und Denkmuster anderer Lebenswelten hineinfühlen und hineinversetzen können.

- Rollendistanz: sich selbst und die eigenen Handlungen auf einer Metaebene von außen betrachten und reflektieren können.

- Metakommunikation: die Aspekte und den Prozess der interkulturellen Kommunikation erklären sowie Probleme in der Kommunikation thematisieren können.

Als spezifisch für die interkulturelle Kommunikation „bleiben allenfalls Fremdsprachenkenntnisse, kulturspezifisches Wissen sowie die Fähigkeit, eigen-, fremd- und interkulturelle 
auf die dänische Mentalität"

Prozesse sich selbst und anderen plausibel beschreiben und erklären zu können.“ (Bolten 2012: 127)

In der interkulturellen Kommunikation stehen gewohnte Kontexte grundsätzlich zur Verhandlung. Die Fähigkeit, kulturbedingte Prozesse und Phänomene in der Kommunikation zu erklären und darauf aufbauend Anderskulturelles als normal, plausibel und sinnhaft zu akzeptieren, ist eine grundlegende Schlüsselkompetenz, denn „Normalität, Plausibilität und Sinnhaftigkeit sind die entscheidenden Elemente, um eine Lebenswelt als „eigene“ anerkennen zu können. Sie werden von den Mitgliedern einer Kultur permanent kommunikativ bestimmt.“ (Bolten 2012: 45)

Wenn unterschiedliche Lebenswelten aufeinandertreffen - sei es innerhalb einer nationalsprachlichen oder zwischen zwei nationalen Kulturen - kann es zu Unterschieden in den kulturgebundenen Auffassungen von Normalität, Plausibilität und Sinnhaftigkeit kommen. Das, was für eine Lebenswelt als normal, plausibel und sinnvoll gilt und als solches nicht hinterfragt wird, kann in einer anderen Lebenswelt gegen bewährte Normen und gängige Normalitätsauffassungen verstoßen.

Jedes interkulturelle Handeln schließt ein, dass die Beteiligten mit anderen, mehr oder minder fremden Konventionen, Normalitätsannahmen und Weltsichten ihrer jeweiligen Partner konfrontiert werden. In der Regel wird eine gemeinsame und in diesem Sinn „dritte“ Handlungsgrundlage im Prozess des Zusammenarbeitens oder -lebens permanent ausgehandelt und korrigiert.

(Bolten 2012: 113)

Eine deutsch-dänische Handlungskompetenz für die interkulturelle Kommunikation definieren wir auf dieser Grundlage als sprachliches und kulturelles Wissen darüber, wie man in bestimmten Situationen in Deutschland und Dänemark die individuellen Schlüsselkompetenzen so einsetzt, dass das eigene Handeln für diese Situationen funktional angemessen ist und deshalb zum Erfolg führt. Als Schlüsselkompetenzen gelten u. a. sprachliche, soziale, kulturelle, fachliche und strategische Fähigkeiten sowie persönliche Eigenschaften wie Empathie, Rollendistanz und eine metakommunikative Kompetenz. Dazu gehört ebenfalls das Kennen von deutschen und dänischen Denk- und Handlungsmustern sowie das Vergleichen- und Umsetzen-Können dieser Muster in der jeweiligen Interkultur in Übereinstimmung mit den für die konkrete Situation geltenden Normen, Konventionen, Normalitätsauffassungen und Erwartungshaltungen. Das Konzept der funktionalen Angemessenheit und seine Anwendbarkeit bei der Erstellung der SMiK-Ratgeber stellen wir im nächsten Abschnitt dar.

\section{Funktionale Angemessenheit als Konzept für die interkulturelle Kommunikation}

\subsection{Funktionale Angemessenheit in der didaktischen Sprachkritik}

Das Konzept der funktionalen Angemessenheit, das wir aus der linguistischen Sprachkritik (cf. Kilian/Niehr/Schiewe 2010, 2013a, 2013b) übernehmen, bezieht sich in der bisherigen Forschung primär auf den Sprachgebrauch. 
Als funktional angemessen gilt ein Sprachhandeln dann, wenn unter Berücksichtigung bestehender Normen und der spezifischen Bedingungen der Kommunikationssituation die sprachlichen Mittel so eingesetzt werden, dass die Wahrscheinlichkeit, das Handlungsziel zu erreichen, optimiert wird.

(Kilian/Niehr/Schiewe 2013a: 298)

Angemessenheit bezieht sich demnach auf die funktional angemessene Einhaltung von Normen und Erwartungshaltungen im Sprachgebrauch. Da sich die Sprachkritik insbesondere mit (intra)sprachlichen Phänomenen (jedoch mit der Voraussetzung einer inneren Mehrsprachigkeit) befasst hat, umfasst die bisherige Auseinandersetzung mit Normen und Erwartungshaltungen bevorzugt sprachliche Phänomene an der Schnittstelle zwischen Interaktion (Mikrokontext), sozialer Situation (Mesokontext) und Kultur (Makrokontext). Es handelt sich insbesondere um (cf. Kilian/Niehr/Schiewe 2010: 41):

- den angemessenen Sprachgebrauch (u. a. Grammatik, Wortwahl und Bedeutung, Stil und Textmuster);

- den angemessenen Umgang mit inhaltlichen und formellen ästhetischen Normen (u. a. Klarheit, Folgerichtigkeit, Wohlgeformtheit etc.);

- den angemessener Einsatz parasprachlicher (Lautstärke, Intonation, Sprechgeschwindigkeit etc.) und nichtsprachlicher (Mimik, Gestik etc.) Phänomene;

- die angemessene inhaltliche, formelle und pragmatische Gestaltung im Hinblick auf die Variablen der Kommunikationssituation (die Intention des Senders und die Erwartungen und Voraussetzungen der Empfänger, die Möglichkeiten, des Textgegenstandes und inhaltes, des Mediums etc.);

- die „Angemessenheit hinsichtlich der konditionellen Relevanz“ (Arendt/Kiesendahl 2013: 347) in der Interaktion.

Wir folgen den grundsätzlichen Voraussetzungen und Maßstäben der linguistischen Sprachkritik, wie sie bei Kilian/Niehr/Schiewe (2010: 52-54) differenziert werden. Insbesondere die Auffassung von und der Umgang der Sprachkritik mit Normen ist für ihre Anwendbarkeit in der Kommunikation an der Schnittstelle zwischen zwei/mehreren Sprachen und Kulturen relevant:

Sprachkritik setzt keine Normen, sondern sie reflektiert Normen, macht sie bewusst und sucht bei Normkonflikten eine Orientierung zu geben.

(Kilian/Niehr/Schiewe 2010: 53)

Normen sind stets kulturell bedingte Phänomene. Missverständnisse entstehen oft, weil Normen aus einer Lebenswelt in einer anderen Lebenswelt entweder unbekannt sind oder die Normvorstellungen der Lebenswelten miteinander in Konflikt stehen (Normenkonflikt). Auch wenn der dänischen und der deutschen Kultur recht viele Gemeinsamkeiten unterstellt werden, deuten existierende Untersuchungen dennoch darauf hin, dass insbesondere in der interkulturellen Geschäftskommunikation zwischen Deutschland und Dänemark immer noch Missverständnisse auf Grund verschiedener stereotyper Vorstellungen von Normen und - aus Normenvorstellungen abgeleitet - von Normalität entstehen. Diese Vorstellungen entstehen durch konventionelle (stereotype) sprachlich-kulturelle Handlungsmuster in den Lebenswelten und als Weltansichten prägen sie die sprachlichen und kulturellen Kompetenzen der Sprecher. 
auf die dänische Mentalität“"

Die Relation zwischen sozialen Strukturen, Kultur und Sprache gehört zu den wesentlichen Ausgangspunkten der Sprachkritik. Des Weiteren korreliert die Auffassung der Sprachkritik von einer grundsätzlichen inneren Mehrsprachigkeit von Einzelsprachen mit „Varietäten, die eigene funktionale Sprachformen und Stile ausprägen“ (Kilian/Niehr/Schiewe 2010: 53), mit der oben beschriebenen Annahme einer Mehrkulturalität. Die Kombination der inneren und äußeren (intra- und interlingualen) Mehrsprachigkeit und (intra- und interkulturellen) Mehrkulturalität ermöglicht einen Analyseansatz, mit dem im Kontext der deutsch-dänischen Kommunikation aus den Interviewdaten eine Orientierung für die (interkulturelle) Kommunikation erstellt werden kann.

\subsection{Analyseverfahren für die funktionale Angemessenheit in der deutsch-dänischen Kommunikation}

Die Ausgangsebene einer Analyse der funktionellen Angemessenheit in der Kommunikation bildet stets der Sprachgebrauch. Die Bezugsebene für die Analyse ist die Ebene der Normen im deutschen und dänischen Sprach- und Kultursystem und die entsprechenden Normalitätsvorstellungen der Sprecher (Sprach- und Kulturkompetenz), auf denen die Beschreibung des Gebrauchs basiert. Abbildung 1 zeigt diesen Zuordnungsrahmen des SMiK-Projekts auf der Ebene des Kultur- und Sprachsystems, des Gebrauchs und der Sprach- und Kulturkompetenz (cf. Hallsteinsdóttir 2013; Hallsteinsdóttir/Farø 2010; Kilian 2015):

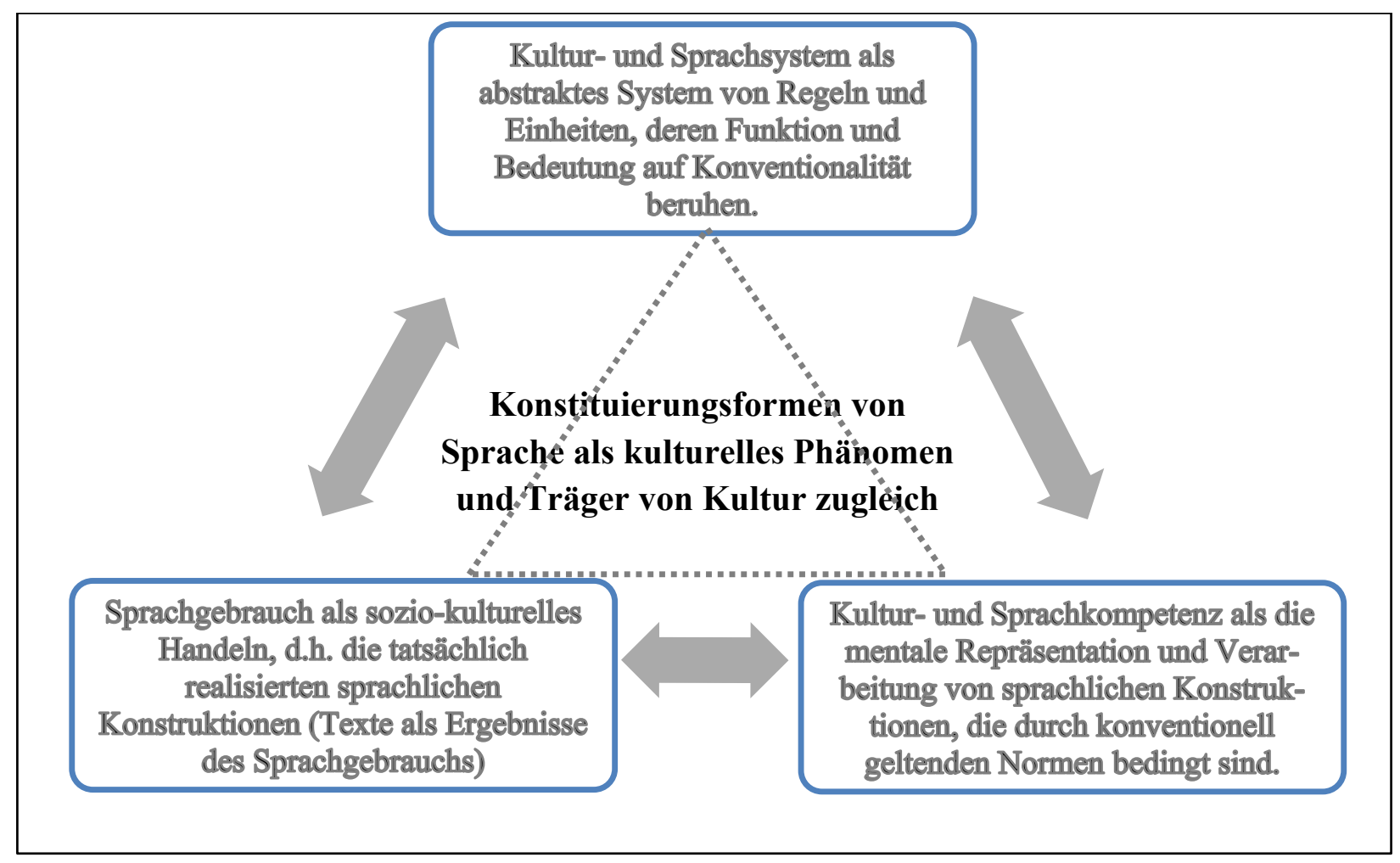

Abbildung 1: Konstituierungsformen von Sprache und Kultur

Ein Analyseverfahren für die funktionale Angemessenheit in der deutsch-dänischen Kommunikation leiten wir aus der Schrittfolge des Analysemodells von Kilian/Niehr/Schiewe (2013b: 311-312) ab. Die Schritte des Modells haben wir an die komplexe Beschaffenheit der Inter- 
viewdaten als metasprachliche Thematisierungen von Sprache und Kultur in der deutschdänischen Zusammenarbeit inhaltlich angepasst:

1. Wahrnehmung eines auffälligen Sprachgebrauchs über Phänomene aus der eigenen oder einer anderen Kultur und Zuordnung als Sprachgebrauch über Abweichungen von Normen, Konflikte mit Normen oder Normenzweifelsfälle. Als auffällig wurde zunächst jede (metasprachliche) Thematisierung von Sprach- und Handlungsgewohnheiten wahrgenommen - auch die, die sich auf die eigene Kultur- und Sprachgemeinschaft beziehen. Alle Aussagen der Interviews, die sich auf sprachliche und Kulturelle Handlungsmuster beziehen, haben den deutsch-dänischen Vergleich als Kontext, und somit enthält eine Eigencharakterisierung auch eine Aussage, die das Anderssein thematisiert (cf. Abschnitt 2.2).

2. Beschreibung des besprochenen Phänomens im Rahmen seines ko- und kontextuellen (situativen) Vorkommens im deutsch-dänischen Kontext (Sach- und Inhaltsebene, Beziehungsebene, Ebene der Situation).

3. Kategoriale und terminologische Verortung des Phänomens in der eigenen Kultur, das die Wahrnehmung in der anderen Kultur als Normabweichung, Normenkonflikt oder Normenzweifel verursacht.

4. Prüfung des Vorkommens des Sprachgebrauchs zum Phänomen in weiteren Texten im Interviewkorpus (ggf. ergänzt durch individuelles sprachlich-kulturelles Wissen sowie in der Forschungsliteratur vorhandene Ergebnisse aus anderen Analysen).

5. Prüfung des Vorkommens des Sprachgebrauchs zum Phänomen in den Fragebogendaten aus dem SMiK-Projekt (cf. Hallsteinsdóttir 2015; Hallsteinsdóttir/Müller 2016; Hofmann/Hallsteinsdóttir in diesem Heft).

6. Beschreibung der Normabweichung, des Normenkonflikts, des Normenzweifelfalls mit Bezug zur Norm der eigenen Kultur.

7. Erklärung der Normabweichung, des Normenkonflikts, des Normenzweifelfalls durch einen Vergleich der eigenen und der fremden Kultur.

8. Formulierung einer linguistisch begründeten sprachkritischen Bewertung und Beurteilung des Sprachgebrauchs aus Schritt 1 und 2 auf der Grundlage der Befunde aus den Schritten 3 bis 7 aus der Perspektive der funktionalen Angemessenheit und Einordnung in das Modell zur situativen Differenzierung des Kontextes (cf. das Modell in Abbildung 2).

9. Generalisierung einer linguistisch begründeten sprachkritischen Bewertung mit Formulierung von allgemeinen Handlungsanweisungen für angemessenes Handeln in konkreten Situationen und der Anfertigung von Illustrationen in Zusammenarbeit mit einer professionellen Graphikerin.

Als Leitfrage für die Analyseschritte steht die Frage danach, wo es im Gebrauch der deutschen oder dänischen Sprache und Kultur Abweichungen von den Erwartungshaltungen, Normalitätsauffassungen und Normen der jeweils anderen Sprache und Kultur gibt, die möglicherweise zu Missverständnissen oder sogar zum Misslingen der deutsch-dänischen Kommunikation führen können. Für die Implementierung der Analyseergebnisse in den Ratgebermaterialien verwenden wir ein Modell, mit dem der jeweilige Kommunikationskontext in Relation zur konkreten Situation differenziert werden kann. 


\subsection{Modell zur situativen Differenzierung des Kontextes}

In ihrem Ansatz, funktionale Angemessenheit zur Bewertung von Gesprächen und Lehrwerken einzusetzen, entwickeln Arendt/Kiesendahl (2013: 346) ein Modell zur Differenzierung des Kontextes. In ihrem Modell erweitern sie die bisher übliche Gliederung des Kontextes in Interaktion (,Mikrokontext"), soziale Situation (,Mesokontext") und Kultur (,Makrokontext") um die Ebene der Interaktionsphase/-sequenz (,Nanokontext"). Die hinzugefügte Ebene enthält ,,[d]as lokal relevante Aktionsgeschehen, das die Relation der Äußerung zu ihrem sequentiellen Kontext in einer turn-by-turn-Perspektive fokussiert" (Arendt/Kiesendahl 2013: 346). Für die interlinguale und interkulturelle Kommunikation erweitern wir dieses Modell um die Ebene der Organisationen und die Ebene der sprachlichen und nicht-sprachlichen Zeichen (cf. Abbildung 2). Die äußeren drei Ebenen umfassen die Phänomene, die die Menschen in einer Situation bedingen. Auf den weiteren drei Ebenen wird die Interaktion zwischen den Menschen in einer Situation erfasst.

Die Mehrschichtenform des Modells soll zeigen, dass die Phänomene der inneren Ebenen von den Phänomenen der umschließenden Ebenen beeinflusst werden. Die äußeren Ebenen halten existente oder kodifizierte Nomen/Kriterien für die funktionale Angemessenheit für den Gebrauch der sprachlichen und nicht-sprachlichen Zeichen auf der jeweils eingeschlossenen Ebene bereit. Die gegenseitige Einwirkung der Ebenen geht in beide Richtungen, denn die inneren Ebenen können ebenfalls nach außen hin einen Einfluss auf die Phänomene der übergeordneten Ebenen ausüben.

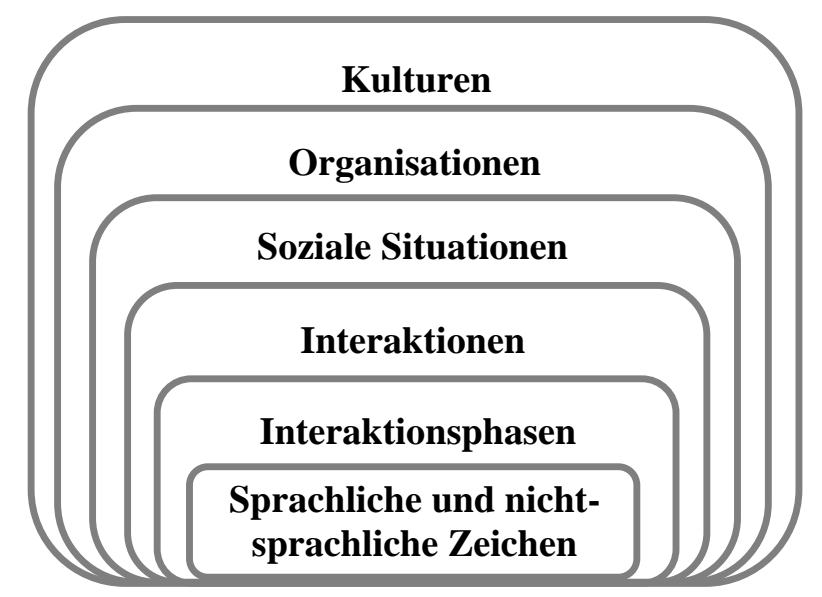

Abbildung 2: Erweitertes Modell zur Differenzierung des Kontextes in der interkulturellen Kommunikation

Die Phänomene, die in den Interviewdaten in den (metasprachlichen) Thematisierungen von Sprach- und Handlungsgewohnheiten als auffällig wahrgenommen werden, können in diesem erweiterten Modell als relevante Variablen einer Situation auf fünf Ebenen eingeordnet werden (cf. hierzu auch Hallsteinsdóttir et al. 2015). Das Modell dient demnach zur Einordnung von (stereotypen) Denk- und Handlungsmustern, Erwartungshaltungen und Normalitätsauffassungen in den Weltansichten, die als unsere Kulturbrille fungieren und zu den Lebenswelten der Menschen gehören. Mit der Einordnung auf den Ebenen des Modells können wir differenziert für konkrete Kontexte aufzeigen, durch welche Phänomene potenzielle sprachlichkulturell bedingte Missverständnisse entstehen können. Zu den Ebenen gehört zudem das Wissen darüber, dass man die eigene Kommunikation an die sprachlichen, sozialen und kultu- 
rellen Bedingungen sowie an die Voraussetzungen der anderen Teilnehmenden in einer Situation anpassen kann.

Das übergeordnete Ziel der Modellierung ist es, herauszufinden, welche kulturellen und sprachlichen Phänomene in der deutsch-dänischen interkulturellen Kommunikation eine Rolle spielen, und diese so zu beschreiben, dass sich die Benutzerinnen und Benutzer der Ratgeber auf eine konkrete, vorgegebene Situation mit ihren Variablen vorbereiten können. Wir setzen dabei voraus, dass, wenn die Benutzerinnen und Benutzer definieren können, welche Variablen für eine Situation relevant sind sowie welche Handlungsmuster mit diesen Variablen verbunden sind, sie dann auch entscheiden können, welche der möglichen Handlungsmuster für diese konkrete Situation angemessen sind.

Die Ebene der Kulturen beinhaltet die intra- und mehrkulturellen Lebenswelten, die durch die Menschen in einer (interkulturellen) Situation repräsentiert sind. Diese Ebene wird als ein offenes System der Lebenswelten aufgefasst, die mit ihren Weltansichten eine konkrete Situation beeinflussen können. Die interkulturelle Kommunikation wird hier somit als die Akzeptanz und Integration von anderen Kulturen in die eigenen Kulturen im Sinne von der Entstehung von (innerer) Mehrkulturalität (cf. Abschnitt 2.3) verstanden und nicht nur als ein interkulturelles Handeln zwischen abgeschlossenen Systemen von zwei oder mehr fremden und/oder nationalen Kulturen betrachtet. Zur Ebene der Organisationen gehören die institutionellen Formen und Strukturen, die für die Konstituierung einer Situation wichtig sind. Solche organisatorischen Phänomene sind häufig kulturspezifisch und sie hängen stark von gesellschaftlichen Strukturen und institutionellen Vorgaben ab. Daher beeinflussen sie auch die soziale Situation eines Kontextes. Mit der sozialen Situation werden die gesellschaftlichen Verhältnisse, in denen sich die Menschen in einer Situation befinden, sowie ihre sozialen Rollen und Beziehungen zueinander zusammengefasst. Die Ebene der Interaktion umfasst den Umgang der Menschen in einer Situation miteinander. Die Interaktion besteht aus Interaktionsphasen, d. h. den einzelnen Teilabschnitten des Handelns in einer konkreten Situation. Auf der Ebene der sprachlichen und nicht-sprachlichen Zeichen werden die in einer Situation verwendeten sprachlichen und non-verbalen Zeichen erfasst, denen Bedeutungen zugeordnet werden. Auf dieser Ebene werden demnach die Zeichen beschrieben, mit denen die Menschen in einer Situation ihre Inhalte, Intentionen, Wünsche etc. kommunizieren.

Die interkulturelle Kommunikation findet, wie oben dargestellt wurde, immer zwischen echten Menschen (in ihrer ganzen Vielfältigkeit) in einer konkreten Situation statt. Demnach ist jede Kommunikationssituation an sich individuell. Es ist aber davon auszugehen, dass das Verhalten der einzelnen Menschen durch (stereotype) sprachliche und kulturelle Denk- und Handlungsmuster der eigenen Lebenswelten geprägt ist. Wir zeigen im Folgenden, wie ausgewählte Thematisierungen von Sprach- und Handlungsgewohnheiten aus der deutschdänischen Kommunikation, die wir in Interviewdaten als auffällige Phänomene wahrgenommen haben, in das Modell zur Differenzierung des Kontextes eingeordnet werden können. 
auf die dänische Mentalität“"

\section{Kommunikationskontexte: Beispiele aus der deutsch-dänischen Kommunikation}

\subsection{Datengrundlage}

Im Frühjahr 2013 wurden 41 deutsche und dänische Personen aus Unternehmen, Projekten und Kulturinstitutionen aus der deutsch-dänischen Grenzregion interviewt. In den Interviews wurden sie nach ihrer Arbeit, ihren interkulturellen Erfahrungen und zur Kommunikation im deutsch-dänischen Kontext gefragt. Die Interviewten sind Firmeninhaber/-innen, Kommunikationsmitarbeiter/-innen oder Mitarbeiter/-innen auf der Leitungsebene, was jedoch bei der Zielgruppe (kleine und mittelständischen Unternehmen, im Folgenden KMU) gelegentlich dieselbe Person ist. Die Interviews fanden vor Ort in den Unternehmen und Institutionen statt, sie dauerten von 12 Minuten bis zu einer Stunde mit einem Durchschnitt von etwa 40 Minuten und sie wurden digital aufgezeichnet. Es wurde nur ein Interview pro Unternehmen durchgeführt (ausführlich dazu cf. Müller 2016).

Mit der Interviewbefragung sollte im Hinblick auf die im SMiK-Projekt zu erstellenden Ratgeber- und Unterrichtsmaterialien der konkrete Bedarf an Ratgebermaterialien und Hilfsmitteln für die deutsch-dänische Kommunikation geklärt werden. Ein wichtiges Ziel dabei war, potenzielle interkulturelle (und andere) Problemstellungen in der deutsch-dänischen Kommunikation aufzudecken. Ein entscheidendes Kriterium für die Auswahl der Interviewten war daher, neben der geographischen Lage in der deutsch-dänischen Interreg-Region, dass sie bereits eigene Erfahrungen mit deutsch-dänischer interkultureller Kommunikation gemacht hatten. Kontakt zum jeweils anderen Markt sollte dabei entweder bereits bestehen oder konkret angestrebt sein. Der Fokus der Untersuchung lag auf Unternehmen mit 50 und weniger Mitarbeitern aus Branchen, die in der der Interreg-Region stark vertreten sind: Tourismus/Erlebnis, Wissen und Kommunikation, Lebensmittel samt Produktion und Handwerk.

In den Interviews, die als dynamische semi-strukturierte qualitative Interviews einzuordnen sind (cf. Kvale 1997), wurde ein im Voraus erarbeiteter Leitfaden angewendet. Der Leitfaden enthielt Fragen zu den Themen Arbeitsaufgaben, Sprachkenntnisse und Sprachstrategien, Kommunikationssituationen, Ausbildung, Netzwerk und Erfahrungen mit interkulturellen Unterschieden. Die Befragten wurden offen zu ihrer Lebenswelt befragt (cf. Bolten 2012; „Livsverden“ Kvale 1997: 111). Mit Fragen zum Alltag sowie zu den Strategien und Ressourcen der Teilnehmer, hierunter auch zu ihrem informellen Wissen, wie z. B. den persönlichen Netzwerken oder dem kulturellen Hintergrund, sollte ein besseres Verständnis des täglichen Arbeitens in der deutsch-dänischen interkulturellen Kommunikation erreicht werden. ${ }^{5}$ Bei der Erstellung des Interview-Leitfadens wurden u. a. Berichte und Literatur zu KMU, deutschdänischen Erfahrungen und Sprache als Ressource bzw. Kompetenz in der Wirtschaft berücksichtigt (cf. u. a. ELAN-Studie 2006; KMU-Studie 2003; List/Wagner 1992; Nordhaug/Grønhaug 1994; PIMLICO-Projektbericht 2011; Rehrmann-Jørgensen/von der Banck 2011; Verstraete-Hansen 2008).

\footnotetext{
${ }^{5}$ Die Ergebnisse der Datenauswertung wurden in Ratgebern zur deutsch-dänischen Kommunikation umgesetzt (cf. Hallsteinsdóttir/Müller 2015a, 2015b, 2015c, 2015d).
} 
Die Interviews wurden von studentischen Hilfskräften ${ }^{6}$ transkribiert und für die wissenschaftliche Auswertung intern katalogisiert (cf. Müller 2016). Für die Ausarbeitung von Ratgebermaterialien wurden die Daten mit den oben dargestellten Analyseschritten ausgewertet und in das Modell zur situativen Differenzierung des Kontextes (cf. Abschnitt 3.3) eingeordnet. In den folgenden Abschnitten zeigen wir exemplarisch, wie wir die Analyse und Einordnung vorgenommen haben.

\subsection{Analysevorgang}

Den neun Schritten des Analyseverfahrens für funktionale Angemessenheit folgend (cf. Abschnitt 3.2) wurden die transkribierten Interviews ausgewertet. ${ }^{7}$ Als Leitfrage für die Analyse stand die Frage nach den von den Interviewten thematisierten Abweichungen von den Erwartungshaltungen, Normalitätsauffassungen und Normen der anderen Sprache und Kultur in der interkulturellen Kommunikation.

Zuerst wurde jede als auffällig wahrgenommene Thematisierung von Phänomenen aus der eigenen oder einer anderen Kultur zu Normen, Abweichungen von Normen, Konflikten mit Normen oder Normenzweifelfällen festgehalten (Schritt 1). Danach wurden die in den Aussagen vorkommenden Phänomene beschrieben (Schritt 2) und in der deutschen bzw. der dänischen Eigenkultur der Interviewten als Norm bzw. Normalität verortet (Schritt 3). Wenn ein Phänomen in einem Interview als auffällig wahrgenommen wurde, wurde dessen Vorkommen in den anderen Interviews überprüft (Schritt 4). Es hat sich in der Analyse bewährt, diesen Schritt gleich am Anfang, d. h. als zweiten Analyseschritt zu implementieren, um ausschließen zu können, dass eine Thematisierung nur in einem einzelnen Interview vorkommt. Zusätzlich wurden Informationen in existierenden Ratgebern und der Forschungsliteratur eingeholt und das Vorkommen in anderen Untersuchungen des SMiK-Projekts überprüft. In den Kulturbrillen-Ratgebern wurden die Interviewdaten durch Zitate aus den Antworten aus einer Fragebogenuntersuchung des SMiK-Projekts zu Hetero- und Spiegelstereotypen von deutschen und dänischen Firmen mit Kontakt zum jeweils anderen Land ergänzt (Schritt 5). Dann erfolgten ein Vergleich zur deutschen bzw. dänischen Kultur und eine darauf basierende Beschreibung der (potenziellen) Normabweichung, des Normenkonflikts oder Normenzweifelfalls in der deutsch-dänischen Kommunikation (Schritte 6 und 7). Die Ergebnisse aus den Schritten 1-7 bilden die Grundlage für eine begründete (sprach)kritische Bewertung und Beurteilung der thematisierten Sprach- und Handlungsgewohnheiten als potenzielle Störfaktoren in der deutsch-dänischen Kommunikation und die Einordnung in das Modell zur situativen Differenzierung des Kontextes (Schritt 8). Das Ergebnis wird in Form von generalisierten Phänomen-/Handlungsbeschreibungen festgehalten (Schritt 9). Für einige Phänomene wurden

\footnotetext{
${ }^{6}$ Wir danken Ursula da Silva Møller-Hansen, Lene Dreisig Sørensen, Mareike Sulamith Schwartz und Nanna Frisgaard Gunnersen für die Transkription.

${ }^{7}$ Die Auswertung inkl. die Übersetzungen der Beispiele ins Deutsche wurde von Katarina Le Müller vorgenommen und von Erla Hallsteinsdóttir überprüft. Beide Autorinnen sprechen Deutsch und Dänisch auf Muttersprachenniveau und sie besitzen umfassende Kenntnisse der deutschen und der dänischen Kultur.
} 
auf die dänische Mentalität"

außerdem Illustrationen ausgearbeitet. Die Ausarbeitung erfolgte in enger Zusammenarbeit zwischen den Autorinnen und der Graphikerin Mie Nørgaard Mouritsen. ${ }^{8}$

Im Folgenden beziehen wir uns ausschließlich auf die Interviewdaten und illustrieren anhand von ausgewählten Beispielen, wie die Interviewdaten mit den oben beschriebenen modifizierten Analyseschritten bearbeitet und das Ergebnis in den SMiK-Ratgebern umgesetzt wurde. Demnach entfällt in den folgenden Analysen der fünfte Analyseschritt. Der vierte Analyseschritt mit der Prüfung des Vorkommens in den Interviews wurde in der Analyse vorgezogen, um eine parallele Anwendung der anderen Analyseschritte für alle Belege zu ermöglichen (cf. Abschnitt 3.2). In den beiden ersten Abschnitten zeigen wir die Analyseschritte explizit, in den weiteren Abschnitten sehen wir von einer expliziten Auflistung ab, da sich die Schritte aus der Beschreibung ergeben.

Die Reihenfolge der folgenden Abschnitte folgt den Ebenen des Modells zur situativen Differenzierung des Kontextes, eine eindeutige Einordnung ist jedoch nicht immer möglich und/oder sinnvoll, denn einige Phänomene beeinflussen die Kommunikation auf mehr als nur einer Ebene und in einigen der angeführten Beispiele wird mehr als nur ein Phänomen thematisiert.

\subsection{Kommunikationskontexte in der deutsch-dänischen Kommunikation}

\subsubsection{Sprachliche und nicht-sprachliche Zeichen}

In den Interviewdaten wurden mehrere auffällige Thematisierungen von Sprache (als bedeutungstragenden Zeichen) und sprachlichen Handlungsgewohnheiten wahrgenommen, die auf der Ebene der sprachlichen und nicht-sprachlichen Zeichen eingeordnet wurden. Beispielhaft zeigen wir die Analyse zu den Thematisierungen von wörtlicher Übersetzung.

Der im ersten Analyseschritt in den Beispielen 1-3 wahrgenommene Sprachgebrauch bezieht sich auf die direkte (wörtliche) Übertragung aus einer Sprache in eine andere. Im zweiten und dritten Analyseschritt halten wir fest, dass zunächst die entscheidende Rolle der Sprache in der interkulturellen Kommunikation betont und auf die Konsequenzen bzw. die Unmöglichkeit von wörtlichen Übersetzungen hingewiesen wird (Beispiel 1 und 2). Die NichtEinhaltung der (sprachlichen) Norm wird einerseits als unterhaltsam, aber andererseits auch als fehlende Kompetenz interpretiert, was wiederum dazu führen kann, dass die fehlende Sprachkompetenz auf andere Faktoren übertragen und als ein genereller Mangel an Kompetenzen betrachtet wird (cf. Beispiel 3):

1. Altså jeg mener jo sproget er det altafgørende, fordi der er så mange forskelle trods alt; de samme ord, når man overscetter dem ordret har de en helt anden betydning, hvor det virkelig går galt. [Also, ich meine ja, dass die Sprache das alles Entscheidende ist, weil es trotz allem so viele Unterschiede gibt; dieselben Wörter, wenn man sie wörtlich übersetzt, dann haben sie eine ganz andere Bedeutung, wo es wirklich schief geht.] (DK10, 09:48).

\footnotetext{
${ }^{8}$ Mie Nørgaard Mouritsen (mienm@hotmail.com) hat als Graphikerin diese und andere Illustrations- und Gestaltungsaufgaben für das SMiK-Projekt ausgeführt.
} 
2. Ein Däne sagt [...]: „Altså bare fordi du er grim, behøver du ikke at vore frcek" [Also nur weil du hässlich bist, musst du nicht auch noch frech sein]. Da fällt beim Deutschen der Unterkiefer, das ist im Dänischen eine Redewendung, völlig normal [...]. Die Deutschen haben da einen ganz anderen Humor, die können bissige Spitzen nicht ab. (D11, 10:05).

3. De ville skrive, at deres varer kom frem i god behold, og så skrev de: ,Wir werden dafür Sorge tragen dass Ihre Ware in guten Umständen ankommt'. „In guten Umständen“ betyder gravid, så tyskerne undrede sig lidt over, hvad skete der med varerne undervejs. Det er sådan nogle småting, altså de kan vare morsomme, men de kan altså også sige til mig som tysker: „jamen hvis ikke de skriver korrekt tysk/brochurerne er forkert, så er deres varer nok også forkerte “. [Sie wollten schreiben, dass ihre Waren in gutem Zustand ankamen, und dann schrieben sie: „Wir werden dafür Sorge tragen dass Ihre Ware in guten Umständen ankommt“. „In guten Umständen“ bedeutet schwanger, so dass sich die Deutschen etwas darüber wunderten, was unterwegs mit den Waren passiert war. Es sind solche Kleinigkeiten, die auch lustig sein können, aber sie können also auch zu mir als Deutsche sagen: „Also, wenn sie nicht korrekt Deutsch schreiben oder die Prospekte fehlerhaft sind, dann sind ihre Waren sicher auch fehlerhaft."] (DK27, 05:59).

Die Beschreibung aus Analyseschritt 6 und das Ergebnis des Vergleichs aus Schritt 7 zeigen, dass gewohnte Strukturen und sprachliche Normen der Muttersprache in der anderen Sprache als Normenabweichungen oder -zweifelfälle aufgefasst werden, wenn sie direkt übertragen werden. Die wörtliche Übersetzung und die unreflektierte Übernahme von sprachlichen Strukturen und ihren Funktionen im Sprachgebrauch setzt eine Symmetrie in der deutschen und in der dänischen Sprache voraus, die es nicht gibt.

Die Einordnung der hier besprochenen Thematisierungen erfolgte im Analyseschritt acht auf der Ebene der sprachlichen und nicht-sprachlichen Zeichen im Modell (cf. Abbildung 2). Beispiel 3 zeigt jedoch, dass die durch eine sprachliche Normenabweichung erzielte Wirkung u. U. auch dem Thema Professionalität und Seriosität zugeordnet werden könnte, das im Modell auf der Ebene der Interaktion einzuordnen wäre.

Thematisierungen von Sprache, wie sie in den Beispielen 1-3 wahrgenommen werden, werden in den Ratgebern unter dem Phänomen Wörtliche und übertragene Bedeutungen mit folgender Beschreibung zusammengefasst. Diese enthält allgemeine Handlungsanweisungen für den angemessenen Umgang mit wörtlichen Übersetzungen, die auf der im Analyseschritt neun entstandenen sprachkritischen Bewertung basiert:

Vorsicht ist geboten, wenn die Muttersprache direkt in die andere Sprache übertragen wird. Wenn man wörtlich in die andere Sprache übersetzt, können „falsche Freunde“ als Wörter mit ähnlicher Form aber unterschiedlichen Bedeutungen Probleme verursachen: Ein Bayer heißt z. B. auf Dänisch nicht bajer (=Bier) sondern bajrer.

Aber auch beim Verstehen der anderen Sprache kann es zu Missverständnissen kommen. Unter Bedeutungen, die man auf der Grundlage der eigenen Kultur meint, verstehen andere mit ihrer Kulturbrille vielleicht etwas ganz anders.

Deutsche und Dänen haben nicht das gleiche kulturhistorische Wissen. Dies zeigt sich bei Dänen manchmal in einer unreflektierten, für deutsche Ohren ungewöhnlichen oder gar unzulässi- 
Katarina Le Müller und Erla Hallsteinsdóttir: „Man muss sich darauf einlassen,

auf die dänische Mentalität“"

gen Verwendung von historisch belasteten sprachlichen Artefakten, z. B. aus dem Zweiten Weltkrieg (Beispiel: „Arbeit macht frei“).

(Müller/Hallsteinsdóttir 2015b: 22)

Weitere in den Interviewdaten identifizierten Thematisierungen, die auf der Ebene der sprachlichen und nicht-sprachlichen Zeichen eingeordnet wurden, wurden in den Ratgebern unter den Überschriften Umgang mit Sprache, Normen und Normalität, ,Du“ - ,Sie“ in Dänemark und „Du“- ,Sie“ in Deutschland beschrieben.

\subsubsection{Interaktionsphasen}

Auf den Ebenen der Interaktionen und Interaktionsphasen wurden auffällige Thematisierungen von Phänomenen wahrgenommen, die in die Ratgeber als Einsatz von Medien, Humor und Professionalität und Seriosität eingegangen sind. Da die Ebenen des Modells nicht immer scharf getrennt werden können, erfolgte die Einordnung in den Ratgebern z. T. fließend ohne eine Trennung zwischen zwei nebeneinanderliegenden Ebenen. Ebenfalls gibt es Thematisierungen, die auf mehr als nur einer Ebene eingeordnet werden können, wie z. B. Professionalität und Seriosität. Die dazu gehörenden Thematisierungen werden im nächsten Abschnitt besprochen, denn sie können im Modell sowohl auf der Ebene der Interaktionsphasen als auch der Ebene der Interaktionen eingeordnet werden. Die Möglichkeiten und Grenzen des Modells, hierunter die Herausforderung, dass Phänomene auf mehrere Ebenen eingeordnet werden können, wird in der abschließen Diskussion aufgegriffen.

Dass Humor interkulturell unterschiedlich sein kann, oder auch situativ unterschiedlich eingesetzt werden kann, wird von den Interviewten in folgenden Beispielen (4-7) thematisiert:

4. Scetter jeg mig ned med dig for eksempel hvor vi har jo heller aldrig mødt hinanden, og du siger et eller andet sjov tog jeg siger et eller andet sjovt - så kører det på en helt anden måde, så griner vi og kunne allerede finde på at pjatte lidt eller. Så har man fået en forbindelse. Men tyskere imellem er der ikke rigtigt nogen forbindelser. [Wenn wir beide uns zum Beispiel zusammensetzen, ohne dass wir uns vorher getroffen haben und Sie sagen etwas Lustiges und ich sage etwas Lustiges - dann verläuft es auf eine ganz andere Art und Weise, dann lachen wir, und wir könnten sogar auch ein bisschen herumalbern. Dann hat man eine Verbindung aufgebaut. Aber zwischen Deutschen gibt es keine richtigen Verbindungen.] (DK8, 32:30).

5. Jeg sad og udbredte mig på tysk med, „planieren“ og så videre og så brugte jeg det forkert. Så i bilen så tegnede han simpelthen en damptromle, der kører hen over, og sagde så: „, das ist planieren!“ ((griner)). Så de HAR humor også på trods for hvad man ellers tror ikke. Ja, men de har masser af humor! [Ich saß und redete auf Deutsch über ,planieren" und so weiter und dann verwendete ich es falsch. Dann, im Auto, zeichnete er einfach eine Dampfwalze, die drüber fährt, und sagte: „Das ist planieren!“ ((lacht)). Sie HABEN also Humor, obwohl man es sonst nicht glaubt. Ja, aber sie haben massenweise Humor!] (DK13, 45:43).

6. Nogle gange er de helt fantastiske til at få pakket noget humor ind $i$ det de siger, som altså virkelig krcever at du forstår sproget - ikke bare at du kan oversctte, hvad det er de siger, men at du egentlig forstår hvad der er den underliggende betydning. [Manchmal 
sind sie fantastisch gut darin, etwas Humor in das hineinzupacken, was sie sagen, was also wirklich voraussetzt, dass man die Sprache versteht - nicht nur, dass man übersetzen kann, was sie sagen, sondern dass man versteht, was die dahinter steckende Bedeutung ist.] (DK9, 28:18).

7. Jamen de har humor, og jeg tror nogle gange så bliver vi rigtig glade, når vi finder ud af at nu kan vi lave noget humor sammen! Og så tror jeg også, at så går vi nogle gange lidt for langt med sådan dansk frisind, og det vil sige, så ender det egentlig med at vi kommer til at trcede dem lidt over toeerne, fordi at nu kan vi jo godt lave lidt humor. [Aber sie haben Humor und ich glaube, wir freuen uns ab und zu richtig darüber, dass wir zusammen Humor machen können! Und dann glaube ich, dass wir manchmal etwas zu weit gehen mit einer Art dänischem Freigeist und das bedeutet, dass es damit endet, dass wir ihnen auf den Fuß treten, weil es ja jetzt so richtig lustig wird.] (DK9, 28:32).

Das Phänomen Humor wird in den Interviews ${ }^{9}$ ausschließlich aus dänischer Perspektive thematisiert (Analyseschritte 1 und 4). Analyseschritte zwei und drei ergeben folgende ko- und kontextuelle Vorkommensformen und Verortungsversuche der Interviewten. In der dänischen Kommunikation wird Humor aktiv eingesetzt, um eine Verbindung zwischen den Gesprächspartnern aufzubauen (Beispiel 4). Dass es das Stereotyp gibt, Deutsche seien humorlos, wird implizit vorausgesetzt. Es wird einerseits angesprochen (Beispiel 5), aber auch zurückgewiesen mit dem Hinweis auf die eigene Erfahrung aus der deutsch-dänischen Kommunikation, dass Deutsche doch Humor haben, der stark sprachlich verankert sei (Beispiel 6). Es wird auch darauf hingewiesen, dass die Tatsache, dass Deutsche und Dänen Humor haben, nicht dazu verleiten dürfe, dass beide auch den gleichen Humor haben. Vielmehr wird festgestellt, dass der dänische Humor und seine Gebrauchsmuster deutsche Gesprächspartner überfordern könne (Beispiel 7). Für Analyseschritte sechs und sieben haben die Interviewten bereits den Vergleich mit der eigenen Kultur vorgenommen. Sie reflektieren zudem explizit über die unterschiedlichen deutschen und dänischen Normen bezüglich der unterschiedlichen Manifestation und Verwendung (Funktion) von Humor in der Interaktion bzw. in der Interaktionsphase, die in der Kommunikation mit Deutschen zu Irritationen führen können, da sie als unangemessen aufgefasst werden. In den Analyseschritten acht und neun wurden zunächst die Relevanz des Phänomens für die dänische Kommunikation und die unterschiedlichen Funktionen von Humor in der deutschen und der dänischen Kommunikation sowie die noch vorkommende stereotype Vorstellung vom humorlosen Deutschen festgehalten. Thematisierungen von Humor, wie sie in den Beispielen 4-7 wahrgenommen werden, werden in den Ratgebern mit folgender Handlungsanweisung und der Illustration in Abbildung 3 dargestellt:

\footnotetext{
${ }^{9}$ In den anderen Untersuchungen werden Unterschiede in der Funktion und Verwendung von Humor auch von deutschen Teilnehmenden thematisiert (cf. Hallsteinsdóttir/Müller 2015b).
} 


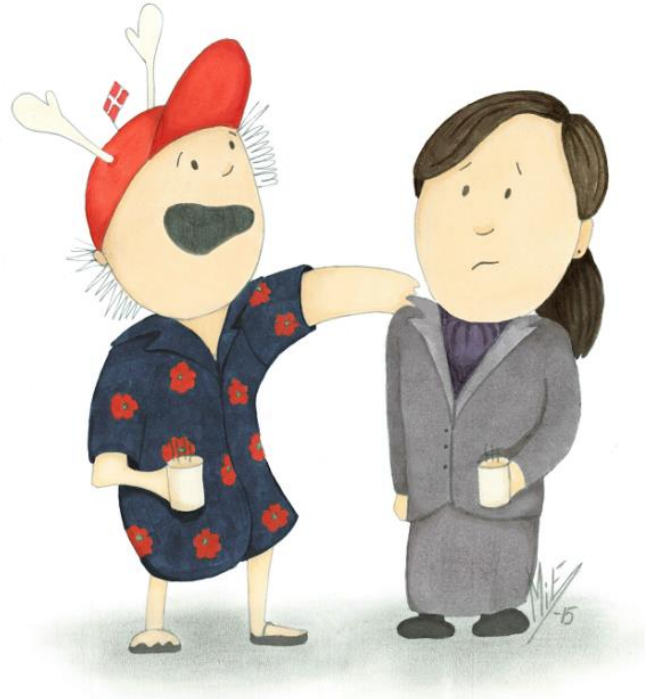

Abbildung 3: Deutsch-dänischer Humor

Humor ist ein wichtiger Teil der dänischen Mentalität und er prägt die Art, wie man in Dänemark kommuniziert.

Die Dänen nutzen ihren Humor auch aktiv zum Aufbau von persönlichen Relationen in der geschäftlichen Kommunikation. Dies ist aus deutscher Sicht nicht immer angemessen - und wird manchmal sogar als unseriös aufgefasst.

Die Dänen stellen beim Kennenlernen gelegentlich etwas erstaunt fest, dass die Deutschen doch Humor haben. Sie beschreiben den deutschen Humor zwar als anders (und manchmal sprachlich schwer zu verstehen) aber nicht unbedingt als schlecht. Die Unterschiede führen dazu, dass viele Dänen den deutschen Humor unterschätzen. Viele Deutsche glauben außerdem selbst, dass die Dänen sie für humorlos halten.

(Müller/Hallsteinsdóttir 2015b: 28)

Bei der Ausarbeitung der Illustration wurden weitere Ergebnisse aus den SMiKUntersuchungen einbezogen, die sich auf die gegenseitige deutsch-dänische Wahrnehmung des für Deutsche bzw. Dänen als angemessen empfundenen Verwendung von Humor und dazugehörigem Verhalten (wie z. B. Entspanntheit, Dresscode, Körperhaltung und gegenseitige Berührung).

\subsubsection{Interaktionen}

Die Ebene der Interaktionen wird in den Ratgebern gemeinsam mit der Ebene der sozialen Situationen beschrieben. Die wahrgenommenen Thematisierungen werden unter den Überschriften Netzwerke und Beziehungen, Professionalität und Seriosität sowie Ordnung muss sein, Informell im Ton, hart in der Sache, Flexibilität oder Regelreiterei?, Umgekehrter Dresscode? und Umgekehrter Dresscode zusammengefasst. Die Thematisierungen von Professionalität und Seriosität können, wie oben dargestellt wurde (cf. Abschnitt 4.3.2), sowohl auf der Ebene der Interaktionsphasen als auch der Ebene der Interaktionen eingeordnet werden. Die folgenden Beispiele gehören zu beiden Ebenen. 
Im Sprachgebrauch in den Beispielen 8-11 werden Unterschiede in Handlungsmustern thematisiert, die für die dänische und deutsche Wahrnehmung von Professionalität und Seriosität relevant sind. Die grundsätzliche Notwendigkeit des professionellen Auftretens in Deutschland wird hervorgehoben, wobei Professionalität und Seriosität an äußeren Merkmalen wie Kleidung, gepflegter Erscheinung, korrektem Auftreten und Pünktlichkeit gemessen wird (Beispiel 8). Implizit liegt der Thematisierung eine Bewertung des für die dänische Kommunikation angemessenen Auftretens als potenziellem Normenkonflikt zugrunde. In den Beispielen wird jedoch klar, dass es eine fließende Grenze zur möglichen Normabweichung, dem Normenkonflikt oder dem Normenzweifelsfall im Hinblick auf die Normen der eigenen Kultur gibt. Die deutsche Pünktlichkeit als die Einhaltung abgesprochener Zeitpunkte wird als ein Ausdruck von Respekt vom Anderen interpretiert (Beispiel 9). Das deutsche Auftreten kann aber auch als zu strukturiert (Beispiel 10) bis hin zu rigide und festgefahren aufgefasst werden, mit der gleichzeitigen Einschätzung, dass die dänische Gemütlichkeit als ein Zeichen für fehlende Kompetenzen oder als unseriös aufgefasst werden kann (Beispiel 11):

8. Hvis du så skal til og markedsføre, skal ind på det tyske marked, så skal man gøre det på en professionel måde. Der mener jeg det hele: også påkloedning, at man er soigneret, at man har korrekt optrceden. Man må ikke ringe og sige „jeg er en halv time forsinket“ fordi børnene skulle $i$ skole eller et eller andet. For tyskerne er alt det der ikke er $i$ planen, det er irritationsmomenter for dem. [Wenn Sie vermarkten wollen, auf den deutschen Markt wollen, dann soll man es auf eine professionelle Art machen. Hier meine ich das Ganze: Auch Kleidung, dass man gepflegt ist, dass man korrekt auftritt. Man darf nicht anrufen und sagen ,ich bin eine halbe Stunde verspätet“, weil die Kinder in die Schule sollten oder so etwas. Für die Deutschen ist all dies nicht mit geplant, es sind Irritationsmomente für sie.] (DK7, 30:00).

9. Man kommer til tiden. [...] Kommer man en halv time før, så bliver man siddende på parkeringspladsen eller $i$ et eller andet skur og venter til klokken er lidt $i$ det aftalte tidspunkt, og så kommer man. Man kommer ikke dappende, for så siger man til den tyske direktør: „Du har jo ikke andet at lave. Vi har godt nok et møde klokken ti, nu er den kvart over ni, jeg kommer alligevel, så du må altså bare have plads til mig “, fordi han har måske noget andet han skal lave indtil klokken 10. Og det virker, det virker forkert, $i k k e$ ? [Man kommt pünktlich [...]. Wenn man eine halbe Stunde zu früh kommt, dann bleibt man im Auto sitzen oder irgendwo anders und wartet, bis es kurz vor dem vereinbarten Zeitpunkt ist, dann kommt man. Man kommt auch nicht reingeschneit, denn dann sagt man dem deutschen Chef: „Sie haben nichts anderes zu tun. Wir haben zwar ein Treffen um zehn Uhr, jetzt ist es viertel nach neun, ich komme trotzdem, Sie müssen sich dann die Zeit für mich nehmen“, weil er vielleicht etwas anderes hat, das er bis $10 \mathrm{Uhr}$ machen soll. Und das wirkt unpassend, nicht?] (DK27, 23:39).

10. Når man afholder et møde mellem to virksomheder, en dansk og en tysk, så kan man vare sikker på at tyskerne de er forberedt-de har struktur og de ved nøjagtigt hvad de vil, de har et mål, de skal gennemgå det. Hvorimod danskerne selvfølgelig også er forberedt, men på en anden måde, tager det sådan lidt mere afslappet, det er ikke så meget: „nu skal vi opnå noget med det her møde" som det er hos tyskerne, og det skaber nogle misforståelser. For tyskere de interpreterer det jo sådan at enten er de ikke gode nok, det er jo kompetencer ikke også, og det er ikke den kompetence vi eftersøger, eller også så 
auf die dänische Mentalität"

synes de „jamen de mener det jo ikke seriøst“. [Wenn man ein Treffen zwischen zwei Unternehmen, einem dänischen und einem deutschen organisiert, dann kann man davon ausgehen, dass sich die Deutschen vorbereitet haben - sie haben Struktur und wissen genau, was sie wollen, sie haben ein Ziel, das sie durcharbeiten sollen. Dagegen haben sich die Dänen natürlich auch vorbereitet, aber auf eine andere Art, sie nehmen die Dinge etwas entspannter, es ist nicht so viel: „Jetzt müssen wir mit diesem Treffen etwas erreichen“, wie bei den Deutschen und dies verursacht einige Missverständnisse. Denn Deutsche interpretieren es ja so, dass sie entweder nicht gut genug sind, es sind ja Kompetenzen, nicht wahr, und es ist nicht die Kompetenz, die wir suchen, oder sie glauben, ,also, sie meinen es ja nicht ernst".] (DK2, 07:55).

11. Die Dänen sehen die Deutschen so, die sind so ,firekantet “ [,rigide"] und da haben sie ja auch Recht. [...]. Das geht so und da geht kein Schritt nach links und rechts. Und der Deutsche sieht den Dänen oft also in Geschäftsbeziehungen und so, dass der sagt: „,Ja, der Däne ist ja ganz gemütlich, aber in Geschäftsbeziehungen geht das nicht mit dem Gemütlichen, das dauert einfach zu lange und das ist ja unprofessionell. "(D12, 35:40).

Auf der Ebene der Interaktion zeigt sich, dass unterschiedliche Interpretationen von Handlungsmustern zu stereotypen Eigenschaftenzuordnungen und normbasierten Wahrnehmungen von Verhalten als unangemessen führen können. Dies bedeutet, dass Handlungen und Erscheinungsformen, die in der einen Kultur als angemessen betrachtet werden, in der anderen Kultur einen Normenzweifel oder Normenkonflikt mit sich führen oder als Normabweichungen eingeordnet werden. Die Befunde aus den Analyseschritten drei bis sieben zeigen, dass es für bestimmte Situationen Unterschiede in der Einordnung von deutsch-dänischen Handlungsmustern gibt, die in der Kommunikation zu Missverständnissen führen können. Für die Ratgeber wurden folgende Handlungsanweisungen als Grundlage für angemessenes Handeln bzw. Reagieren auf die kulturbestimmte Wahrnehmung von Professionalität und Seriosität ausgearbeitet. Die Illustrationen in Abbildung 4 und 5 ergänzen die Beschreibung:

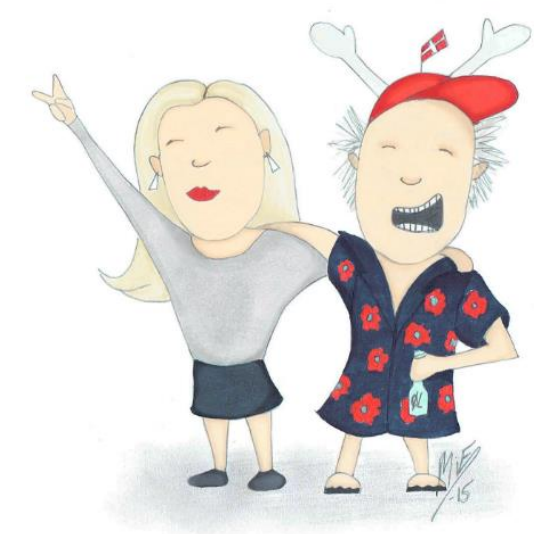

Abbildung 4: Stereotype Auffassung von Deutschen über Dänen 


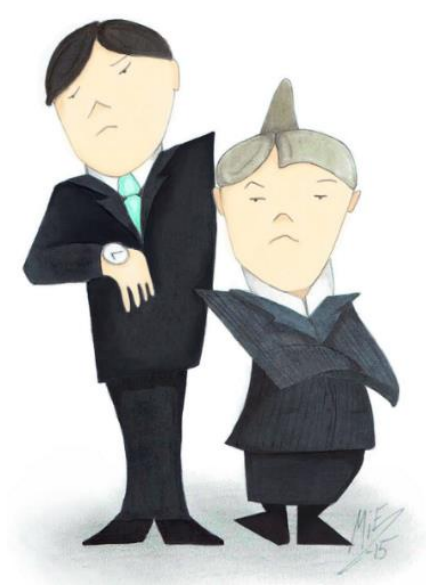

Abbildung 5: Stereotype Auffassung von Dänen über Deutsche

Es gibt kulturgeprägte deutsch-dänische Unterschiede in der Auffassung davon, was Professionalität und Seriosität ausmacht. Deutsche Erwartungshaltungen an Professionalität beinhalten eine gründliche Vorbereitung, einen vollständigen Überblick, die Einhaltung von Vorschriften, effiziente Arbeitsweise und Fokus auf die Arbeitsinhalte - Dienst ist Dienst und Schnaps ist Schnaps.

Was aus deutscher Sicht selbstverständlich als gründliche Vorbereitung aufgefasst wird, wird oft aus dänischer Sicht eher als optional gesehen.

Die Ziele und Ergebnisse eines Treffens verstehen Dänen häufig als einen Prozess, der während des Treffens entsteht und im Verlauf zu Ergebnissen führt. Daher wirken dänische Treffen für Deutsche häufig unstrukturiert und ziellos - oder sie werden positiv gesehen und als flexibel und kreativ erlebt.

(Müller/Hallsteinsdóttir 2015b: 32)

In den Illustrationen wird die Relation zwischen Auto-, Hetero- und Spiegelstereotypen aufgegriffen (cf. Abschnitt 2.2). Damit soll zusätzlich verdeutlicht werden, dass die die eigene Selbst-Wahrnehmung nicht unbedingt mit der Art und Weise übereinstimmt, wie man von anderen wahrgenommen wird.

\subsubsection{Soziale Situationen}

Wie oben (cf. Abschnitt 4.3.2) dargestellt wurde, gehören die Thematisierungen unter den beiden Überschriften zu Dresscode zur Ebene der sozialen Situationen während sich die anderen Thematisierungen mit der Ebene der Interaktionen überschneiden. Sowohl aus deutscher als auch dänischer Sicht werden unterschiedliche Normen bezüglich Dresscode thematisiert. In diesen Thematisierungen klingen die unterliegende Wert- und Normvorstellungen vom sozialen Status und sozialer Interaktion mit an. Insbesondere für die dänischen Interviewten ist es auffällig, wie die Deutschen zwischen Arbeit und Freizeit unterscheiden. Die Normabweichung aus deutscher Perspektive ist die Nichteinhaltung von professionellen Kleidernormen, und dann die Verwirrung, dass es im Privatleben der Dänen einen Dresscode gibt, der nicht nur aus gemütlicher Freizeitkleidung besteht. Der an äußere Bedingungen angepasste (eher pragmatische) dänische Umgang mit Kleidung wird sowohl aus dänischer (Beispiel 12) als aus deutscher (Beispiel 13) Sicht als ein potenziell als Normverletzung wahrgenom- 
auf die dänische Mentalität“"

menes Phänomen thematisiert. Das als sehr formell wahrgenommene Aussehen der Deutschen im Vergleich zur lockeren dänischen Art wird einerseits in Relation zur sozialen bzw. organisatorischen Hierarchie gesetzt (Beispiel 13 und 14) und andererseits als ein deutschdänischer Unterschied beschrieben, der durch eine als andersartig wahrgenommene Aufteilung zwischen beruflich und privat bzw. Arbeit und Freizeit (Beispiel 15, 16 und 17) entsteht.

12. Vi siger: „lad nu vare med at blive bange når de dusser jer, for de ved ikke bedre “. Og altså de skal ikke tage det fortrydeligt op. De skal heller ikke tage det fortrydeligt op at han måske ikke er sådan helt formelt kloedt men/fordi han er lidt afslappet det er varmt uden for så han har nok ikke noget slips på han har slet ingen jakke på når han er $i$ møde. Og $i$ må gernelog da han jo er vart så må $i$ jo gerne hoenge jeres jakker også for ligesom at åbne lidt for kommunikationen. [Wir sagen: „Erschrecken Sie nicht, wenn sie [die Dänen] Sie duzen, denn sie wissen es nicht besser". Und Sie sollten es ihnen nicht übel nehmen. Sie sollten es ihnen auch nicht übel nehmen, dass sie vielleicht nicht ganz so formell gekleidet sind, weil sie etwas entspannt sind, es ist draußen warm und daher haben sie vermutlich keine Krawatte um und gar keine Jacke, wenn sie zum Treffen sind. Und [...] da sie die Gastgeber sind, dürfen Sie gerne Ihre Jacken ablegen, auch um die Kommunikation ein wenig aufzulockern.] (DK27, 25:01).

13. Dann saß an der Ecke, da saß einer, der sah aus wie so ein Hausmeister, der rannte in seinen Crocks rum und so, mit seiner Arbeitshose und immer, wenn der an mir vorbeiging, dann haute der mir auf die Schulter. Und ich denke immer nur, also was ist das denn für ein Idiot bitte, also bei uns würde keiner auf die Idee kommen, seinen Hausmeister mit in so eine Besprechung zu setzen. Und dann kriegte man aber irgendwann mal mit, [...] eigentlich war der der Chef vom Ganzen. Der hatte nur keine Lust auf Schlips und Anzug. (D11, 17:55).

14. Vi oplever det jo utrolig tit ved de der falles møder vi holder, hvor tyskerne jo altid kommer dresset op i fuldstondig fineste uniform - al sildesalat og hvid skjorte og alt muligt, og vi har jo/for eksempel danske beredskabschefer, som i ansvar og prestige og lønmoessig og alt muligt er langt, langt højere end dem, kommer måske i en t-shirt. [Wir erleben es ja unglaublich häufig in den gemeinsamen Treffen, die wir abhalten, zu denen die Deutschen immer in der absolut feinsten Uniform erscheinen - alle Orden und weißes Hemd und alles, was dazugehört. Und wir haben zum Beispiel dänische Bereitschaftschefs, die im Hinblick auf Verantwortung, Prestige, Gehälter und so weiter, viel, viel höher gestellt sind als sie [die Deutschen]. Sie erscheinen vielleicht in einem T-Shirt.] (DK10, 54:06).

15. De [tyskerne] er meget formelle i deres dagligdag, og det er klart at det formelle går lidt af når man loerer dem at kende på en anden vis, men vi danskere er mere sådan lidt straight, der er ikke nogen stor forskel på os om dagen og om aftenen, og det er noget man skal vaere opmoerksom på. [Sie [die Deutschen] sind sehr formell in ihrem Alltag, und es ist klar, dass das Formelle etwas verschwindet, wenn man sie in anderen Zusammenhängen kennenlernt, aber wir Dänen sind eher irgendwie ein wenig straight, es gibt keinen großen Unterschied zwischen uns am Tag und am Abend, dies ist etwas, auf das man aufmerksam sein sollte.] (DK6, 08:35).

16. Jeg synes jo en tysker er meget - der er - de er på to måder; de er når de arbejder, så er de utrolig dygtige og meget, meget energiske, men ligeså snart de har fri - så hygger de 
sig! Og det er det jeg godt kan lide. [Ich finde ja, dass Deutsche sehr - sie erscheinen auf zweifache Weise; wenn sie arbeiten, dann sind sie unglaublich tüchtig und sehr, sehr energisch, aber sobald sie frei haben - dann machen sie es sich gemütlich! Und das ist das, was ich mag.] (DK14, 21:16).

17. [Über Dänemark] Dieses bei der Party, das fand ich total stressig, dieses Aufbrezeln, das sind Regeln, die brauche ich nicht, ich komme mit einer Jeans auf eine Party, weil ich davon ausgehe. Ich will nicht im Abendkleid dahin kommen und in Stöckelschuhen dann die Runde Handschlag machen. (D14, 41:50).

Auf der Grundlage der als auffällig wahrgenommenen Thematisierungen von Kleidung und Aussehen wurden für die Ratgeber folgende Beschreibungen unter den Überschriften Umgekehrter Dresscode? und Umgekehrter Dresscode ausgearbeitet. Die Illustrationen in Abbildung 6 und 7 ergänzen die Beschreibung mit einer Visualisierung der deutsch-dänischen Heterostereotypen zur Arbeits- und Freizeitbekleidung in Deutschland und Dänemark.

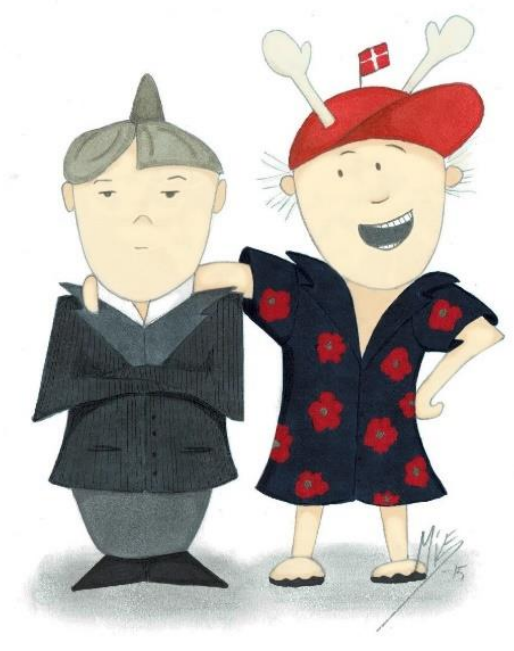

Abbildung 6: Umgekehrter Dresscode?

„Kleider machen Leute“ - An vielen Arbeitsplätzen in Deutschland gilt eine formelle Kleiderordnung. Die Kleidung signalisiert häufig, welche Funktion die Mitarbeiter haben. Durch die dänische Kulturbrille wirkt der deutsche Dresscode oft sehr steif.

Das Nicht-Einhalten des gepflegten Aussehens - Anzug, gebügeltes Hemd, Krawatte und geputzte Schuhe - bei einem Treffen kann in bestimmten Bereichen Ihren deutschen Geschäftspartner irritieren, und im schlimmsten Fall nimmt er Sie nicht ernst. Es gibt aber auch in Deutschland Branchen und Firmen, in denen die Kleiderordnung keine große Rolle spielt - und in Dänemark gibt es ebenfalls Dresscodes in einigen Branchen und Firmen.

(Müller Hallsteinsdóttir 2015b: 36) 


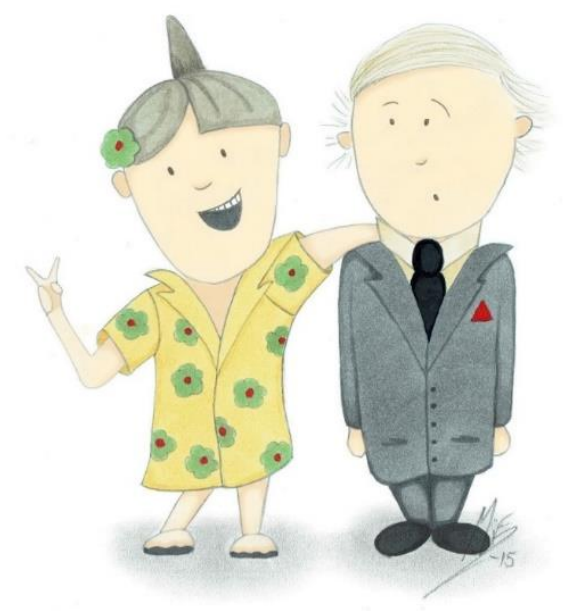

Abbildung 7: Umgekehrter Dresscode

„Kleider machen Leute“ - In Deutschland gilt: Freizeitkleidung für die Freizeit. Die häufige Freizeitkleidung in der Arbeitswelt in Dänemark kann durch die deutsche Kulturbrille als unüblich betrachtet werden. Sie kann zudem auch verwirrend wirken, denn an der Kleidung ist nicht ablesbar, welchen Status jemand hat.

Deutsche in Freizeitkleidung werden dagegen von Dänen oft als „schlecht gekleidet“ empfunden.

In Dänemark gilt eine formelle Kleiderordnung zu vielen privaten Anlässen, denn auch die Dänen tragen Krawatte und Anzug: Zu Festen und beim Feiern wird die feine Garderobe hervorgeholt.

(Müller Hallsteinsdóttir 2015b: 37)

\subsubsection{Organisationen}

Die Ebenen Kulturen und Organisationen werden in den Ratgebern unter einem Kapitel zusammengefasst. Auf der Ebene der Organisationen wurden insbesondere Thematisierungen von Hierarchie als auffällig wahrgenommen. Diese werden unter den Überschriften Attitüde, Sichtbare und unsichtbare Hierarchien und Hierarchische Strukturen präsentiert. Die vorliegende Beleganalyse bezieht sich auf die beiden letzten Überschriften.

Auch wenn die Interviewten unterschiedlich darauf eingehen, können wir feststellen, dass das Phänomen Hierarchie und die dazugehörigen Normvorstellungen stark durch die Gegensätze Individualität versus Gruppenmentalität (Beispiel 18) und Struktur versus Flexibilität (Beispiel 19) geprägt werden. Unterschiedliche organisatorische Traditionen und die zugehörigen Normvorstellungen, werden sowohl von den dänischen als auch den deutschen Teilnehmern thematisiert. Von den deutschen Teilnehmern wird die dänische Lockerheit und Entspanntheit (Beispiel 20), sowie die Gruppenorientierung (Beispiel 18) und die Verschmelzung der dänischen Hierarchieebenen (Beispiel 21) zum Phänomen Hierarchie gezählt. Da Hierarchien nach deutschen und dänischen Normen nicht auf dieselbe Art und Weise markiert werden, kann die als unsichtbar empfundene dänische Hierarchie bei Deutschen eine Unsicherheit im Umgang mit Dänen verursachen (Beispiel 21). Aus dänischer Sicht wird auf die Zähheit der als sehr fest und komplex (Beispiel 22) aufgefassten deutschen Strukturen (Beispiel 19) und 
der in diesem Zusammenhang erlebten fehlenden Bereitschaft eingegangen, selbstständig Verantwortung zu übernehmen (Beispiel 23):

18. In Dänemark ist ja dieses Hierarchieverhalten nicht so stark ausgeprägt. Da arbeitet man als Gruppe und dann ist das okay und in Deutschland ist das wirklich auch, dass du die Hierarchieleiter..., wenn du irgendetwas besprichst, dass du dann genau weißt, an wen du dich wenden musst. (D12, 18:25).

19. Måske alligevel at de skal have det måske serveret på en anden måde - deres opfattelse..., jamen det ved jeg ikke, den er sådan lidt mere - vi er jo mere fleksible og sådan noget de er mere sådan struktur. [Vielleicht doch, dass sie es auf eine andere Art serviert bekommen sollen, ihre Auffassung..., also, das weiß ich nicht, sie ist irgendwie etwas mehr wir sind ja flexibler und Ähnliches und sie sind eher so Struktur.] (DK24, 12:28).

20. In Dänemark ist es relativ einfach, da freut sich der Chef, das findet der ,,hyggeligt“ [,,gemütlich"], mal eben schnell mit dem Verkäufer zu reden und dann ordnet der das und dann ,,das kriegen wir schon hin“ und das geht alles sehr locker über die Bühne [...], dafür ist Deutschland einfach ein bisschen zu steif. (D11, 21:51).

21. Den danske mentalitet er nogle gange, så er vi med af interesse, lederen vil gerne med ned på gulvet og morke hvad der sker, hvad der foregår, „,kan jeg hjoelpe med noget?“ Hvor i Tyskland, der er man lidt mere opmoerksom på at beskytte sin position og autoritet, og vare mere skarp på: hvor deltager man, hvor deltager man ikke. Det er det kan du forvente af mig, det er det du ikke kan forvente af mig. Der bliver tyske ledere, som lige pludselig skal samarbejde med en dansk chef, nogle gange meget usikre på: ,hvad er det egentlig han vil? " [Die dänische Mentalität ist manchmal, da machen wir aus Interesse mit, der Chef möchte gerne zum Fußvolk und miterleben, was passiert: „Kann ich bei etwas helfen?" Dagegen achtet man in Deutschland etwas mehr darauf, seine Position und Autorität zu beschützen, und genauer zu überlegen, wo mache ich mit, was mache ich nicht. Das können Sie von mir erwarten, das können Sie nicht von mir erwarten. Hier werden deutsche Chefs, die auf einmal mit einem dänischen Chef zusammenarbeiten sollen, manchmal sehr unsicher darüber, „was will er eigentlich?“] (DK9, 29:36).

22. Jeg ville gerne have vidst - altså - generelt, så synes jeg at ting tager lang tid $i$ Tyskland. Der er mange led det skal igennem, der er mange, der skal høres. [Generell, finde ich, dass in Deutschland die Dinge viel Zeit brauchen. Man muss durch viele Ebenen und viele müssen gehört werden.] (DK10, 29:56).

23. Det er nemlig også typisk tysk at ikke tage ansvar for noget - fordi at det er ikke mit bord, det er ikke mig. Jeg må ikke noget - det er typisk tysk ikke at tage ansvar. [Es ist nämlich auch typisch deutsch, keine Verantwortung für etwas zu übernehmen, weil es nicht mein Bereich ist, ich bin es nicht, ich darf nichts - es ist typisch deutsch die Verantwortung nicht zu übernehmen.] (DK8, 40:42).

Die Wertung der wahrgenommenen hierarchischen Strukturen fällt bei den Interviewten unterschiedlich aus. Die von einigen als etwas zu steif (Beispiel 20) empfundenen deutschen Strukturen werden auch als positiv empfunden, denn sie erlauben eine genaue Verortung des relevanten Gesprächspartners (Beispiel 18). Hier zeigt sich die individuelle Wertung als ein grundlegendes Element in der Einordnung von Normen bzw. Normenabweichungen: ob 

auf die dänische Mentalität"

Deutsche und Dänen die thematisierten Phänomene oder Verhalten als Normenzweifel oder Normenkonflikt erleben, hängt auch von ihren individuellen Vorstellungen und Vorlieben ab.

Folgende Beschreibungen wurden auf der Grundlage der als auffällig wahrgenommenen Thematisierungen von hierarchischen Strukturen unter den Überschriften Sichtbare und unsichtbare Hierarchien und Hierarchische Strukturen verfasst. Die Visualisierung der deutschdänischen Heterostereotypen zu Hierarchien in Abbildung 8 und 9 ergänzen die Beschreibung.

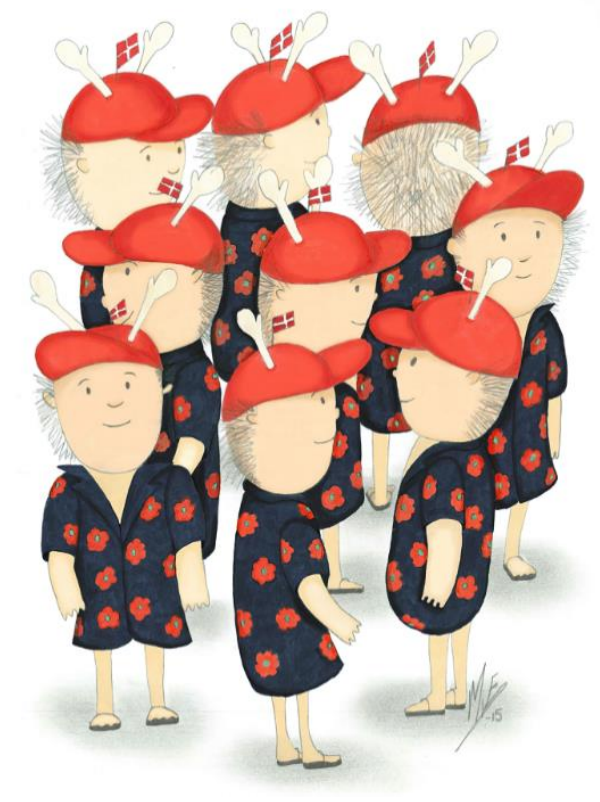

Abbildung 8: Die stereotype deutsche Vorstellung von dänischen Hierarchiestrukturen

Obwohl es aus deutscher Sicht nicht immer zu erkennen ist: Hierarchien gibt es auch in Dänemark. Die hierarchischen Strukturen sind nur nicht immer so sichtbar wie in Deutschland.

$\mathrm{Ob}$ es in einem Unternehmen flache oder hierarchische Strukturen gibt, ist auch eine Frage der jeweiligen organisatorischen Strukturen.

Bewerten Sie das, was Sie in der anderen Kultur als Organisationsstrukturen wahrnehmen, nicht gleich anhand Ihrer eigenen Vorstellung von organisatorischen Strukturen und Verhalten. Fragen Sie sich lieber, welche organisatorischen Strukturen und Handlungsmuster in Ihrer Branche in Deutschland und Dänemark ,normal“ sind und welche Unterschiede und Gemeinsamkeiten es gibt, die die Kommunikation beeinflussen können.

(Müller/Hallsteinsdóttir 2015b: 40) 


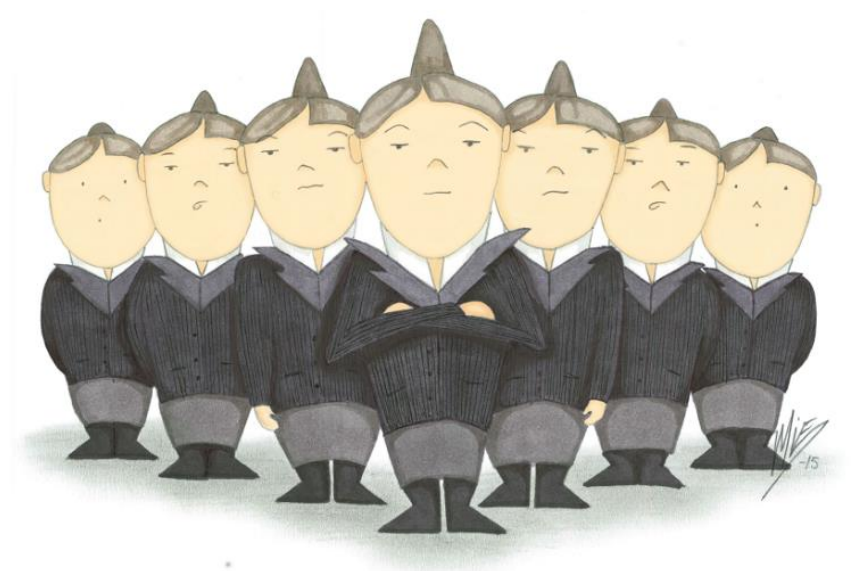

Abbildung 9: Die stereotype dänische Vorstellung von deutschen Hierarchiestrukturen

Die Hierarchiemerkmale und Strukturen sind in deutschen Unternehmen meist deutlich sichtbarer als in Dänemark.

Deutsche Organisationen werden aus dänischer Sicht häufig als kompliziert, steif und langsam empfunden. Die hierarchischen Strukturen der deutschen Organisationskultur können durch die dänische Kulturbrille sogar als die direkte Ausübung von Macht interpretiert und/oder - auf den unteren Stufen der Hierarchie - als fehlender Wille, Verantwortung zu übernehmen.

Die Unsichtbarkeit dänischer Hierarchiestrukturen kann dagegen für Deutsche verwirrend sein und als unprofessionell aufgefasst werden.

(Müller/Hallsteinsdóttir 2015b: 41)

\subsubsection{Kulturen}

Auf der Ebene der Kulturen wurden in den Interviews die thematisierten Phänomene LifeWork-Balance bzw. Arbeitseinstellung/-zeiten sowie Nationalität und Größenverhältnis der Länder als auffällig wahrgenommen und in den Ratgebern unter den Überschriften „Erst die Arbeit - dann das Vergnügen? “ und „,Großes Land - kleines Land“ zusammengefasst. Diese Phänomene wurden markant häufiger von den deutschen als von den dänischen Interviewten angesprochen.

Die Unterschiede in der Arbeitskultur und den dazugehörenden Normvorstellungen werden in den Beispielen 24-26 thematisiert, wobei die deutschen Interviewten die dänische Arbeitskultur positiv als kinderfreundlich (Beispiel 24) und mitarbeiterorientiert (Beispiel 24) bewertet wird. In der deutschen Arbeitskultur, die als Vergleichsbasis fungiert, bekommt die Arbeit eine starke Priorität dem Privaten gegenüber zugeordnet (Beispiel 26) und sie erscheint durch den impliziten Vergleich (Beispiel 24) auch als weniger familienfreundlich:

24. Ich habe [in Dänemark] nie negativ erlebt, weder bei mir noch bei anderen, wenn einer anrief, eine E-Mail schrieb, mein Kind ist krank, ich bleibe zuhause oder wenn auch mal ein Kind mit auf die Arbeit kam, oder Treffen werden nicht nachmittags um halb sechs gelegt. Solche Geschichten, also das ist ja eine völlig andere Arbeitskultur. (D2, 36:30).

25. Also grundsätzlich ist die dänische Kultur, auch was so Kultur am Arbeitsplatz angeht, lockerer, die ist auch mehr mitarbeiterzentriert oder zentriert [...]. Wenn in Deutschland 
Katarina Le Müller und Erla Hallsteinsdóttir: „Man muss sich darauf einlassen,

auf die dänische Mentalität"

ein Arbeitnehmer mit vierzig Grad Fieber zur Arbeit kommt, dann bekommt er einen Orden angehängt: „Ey geil das du trotzdem kommst, hoffentlich überlebst du den Arbeitstag“. In Dänemark sagt der Chef: „,Sieh zu, dass du nach Hause kommst, willst du meine Mitarbeiter anstecken, du bist eine Gefahr für das Unternehmen. “(D8, 32:08).

26. Wenn er sieht, dass ich abends um acht jeden Tag da war, dann will er mich doch irgendwann befördern. In Dänemark würde mein Chef sagen: „Sag mal wirst du mit deiner Arbeit nie fertig? " (D9, 48:59).

Die als auffällig wahrgenommenen Thematisierungen von Arbeitsleben bilden die Grundlage für folgende Beschreibung unter der Überschrift „,Erst die Arbeit - dann das Vergnügen? “ in den Ratgebern. Die Illustration in Abbildung 10 ergänzt die Beschreibung mit einer Visualisierung der stereotypen Vorstellungen zur Arbeitszeit in Deutschland und Dänemark

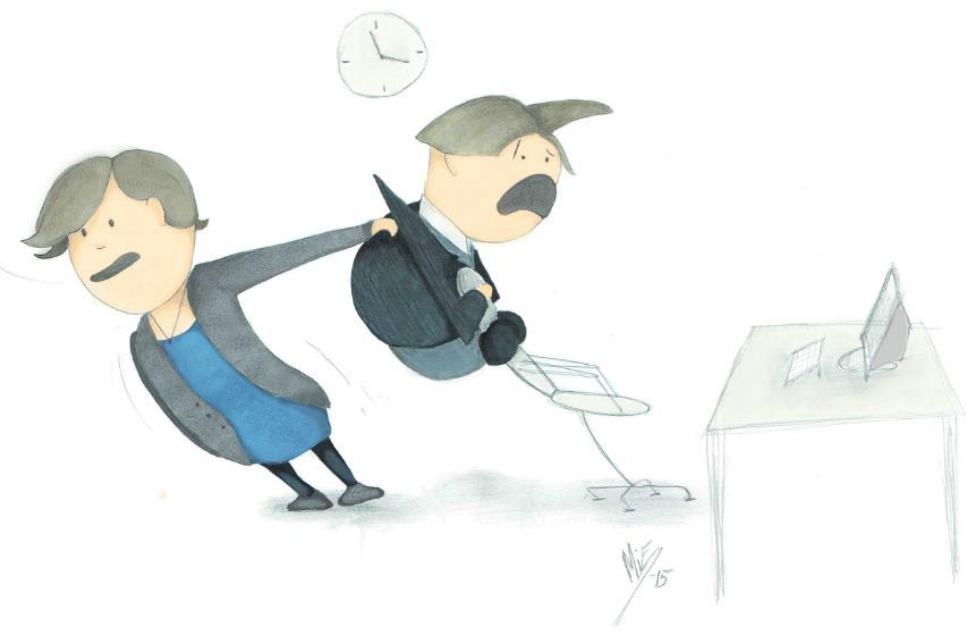

\footnotetext{
Abbildung 10: Stereotype Vorstellungen von deutsch-dänischen Unterschieden im Berufsleben
}

Zwischen Deutschland und Dänemark gibt es unterschiedliche Erwartungen dazu und Auffassungen davon, was als „Normalität“ in der Arbeitskultur angesehen wird. Dänemark hat den Ruf, dass dort eine ausgeglichene Work-Life-Balance zur Normalität gehört. Familie und Freizeit sind in der Tat sehr wichtig. Öffnungszeiten von Institutionen sowie normale Arbeitszeiten sind darauf angelegt, dass der Nachmittag der Familie gehören kann. Dies kann durch die deutsche Kulturbrille als eine Tendenz dazu, „Dienst nach Vorschrift“ zu machen, aufgefasst werden. Trotzdem ist es in einigen Berufen nicht ungewöhnlich, dass man am Abend dann wieder einige Stunden arbeitet. Die deutschen Arbeitszeiten sind anders als in Dänemark und teilweise existiert auch eine andere Kultur mit der Erwartung, dass man der Arbeit eine höhere Priorität einräumt als dem Privaten.

(Müller/Hallsteinsdóttir 2015b: 42)

\subsection{Diskussion}

In der Analyse der Interviewdaten haben wir die im Abschnitt 3.2 vorgestellten, für die Analyse von Interviewdaten zur interkulturellen Kommunikation modifizierte Schrittfolge des Analysemodells von Kilian/Niehr/Schiewe (2013: 311-312) angewendet. Die Grundlage der Analyse bildeten Wahrnehmungen vom auffälligen Sprachgebrauch zu den in den Interviews thematisierten Phänomenen und Handlungsmustern in der deutsch-dänischen Kommunikati- 
on. Im ersten Analyseschritt wurde zunächst jede im Sprachgebrauch der Interviews vorkommende Thematisierung von deutschen und dänischen Sprach- und Handlungsgewohnheiten erfasst. Die Prüfung des Vorkommens des Sprachgebrauchs in weiteren Texten im Interviewkorpus im Analyseschritt vier wurde an den Anfang der Analyse gestellt, um in allen Analysen die kollektive Existenz der wahrgenommenen Thematisierung abzusichern. In den Analyseschritten zwei und drei wurden die Phänomene und Handlungsmuster beschrieben, die die in den Interviews wahrgenommenen Thematisierungen enthalten, und ihre ko- und kontextuelle Zugehörigkeit erfasst. Die Grundlage für diese Analyseschritte bildeten die in den Interviews geäußerten eigenen Erfahrungen und Vorstellungen, die als Thematisierung von Abweichungen von Normen, Konflikten mit Normen oder Normenzweifelsfällen in Situationen im deutsch-dänischen Kontext wahrgenommen wurden. Alle Aussagen der Interviews, die sich auf sprachliche und kulturelle Handlungsmuster beziehen, haben den deutschdänischen Vergleich als Kontext, und somit wurde in Anlehnung an Bolten (2012, cf. auch Abschnitt 2.2) eine Eigencharakterisierung auch als eine Aussage ausgewertet, die das Anderssein thematisiert.

Die Ergänzung der Analyseergebnisse durch das individuelle sprachlich-kulturelle Wissen und in der Forschungsliteratur vorhandene Ergebnisse aus anderen Analysen im Analyseschritt vier sowie die Prüfung des Vorkommens des Sprachgebrauchs zum Phänomen in den Fragebogendaten aus dem SMiK-Projekt im Analyseschritt fünf erfolgten bei der Erstellung der Ratgeber. Da sich dieser Beitrag auf die Auswertung der Interviewdaten konzentriert, wurden diese Analyseschritte hier nicht ausführlich beschrieben. Die Beschreibung der Normabweichung, des Normenkonflikts, des Normenzweifelfalls im Analyseschritt sechs sowie ihre Erklärung im Vergleich zwischen der deutschen und der dänischen Kultur im Analyseschritt sieben erfolgten als eine Zusammenfassung und Ergänzung der Aussagen in den Interviews. Abschließend wurde in Analyseschritten acht und neun auf der Grundlage der Ergebnisse aus den anderen Schritten eine linguistisch begründete sprachkritische Bewertung und Beurteilung auf der Grundlage des Konzepts der funktionalen Angemessenheit vorgenommen. Für die Formulierung von Erklärungen und allgemeinen Handlungsanweisungen für angemessenes Handeln in konkreten Situationen in der deutsch-dänischen Kommunikation wurde eine Einordnung in das Modell zur situativen Differenzierung des Kontextes (Abbildung 2) vorgenommen. Die Handlungsanweisungen wurden abschließend durch Illustrationen ergänzt. Die hier dargestellten Analysen stellen eine Auswahl der für die Ratgeber analysierten und aufbereiteten Interviewdaten dar.

Die Ergebnisse zu den Beispielen 1-3 zeigen, dass es im Umgang mit sprachlichen und nichtsprachlichen Zeichen Unterschiede gibt, die nicht auf abstrakte Mentalitätsunterschiede zurückzuführen sind, sondern auf konkreten, häufig den Sprechern unbewussten Unterschieden im Gebrauch von Bildern, Sprache, Körpersprache etc. basieren. Diese Beispiele verdeutlichen, wie wichtig der funktional angemessene Umgang mit Sprache in der Kommunikation ist, d. h. dass man den Sprachgebrauch funktional an die konkrete Situation und die darin teilnehmenden Kommunizierenden anpasst.

Die Beispiele 4-7 zum Phänomen Humor auf der Ebene der Interaktionsphasen sprechen einerseits ein in Dänemark bekanntes Stereotyp an (Die Deutschen haben keinen Humor), sie wiederlegen andererseits aber auch dieses Stereotyp. Sowohl die Relevanz als auch unter- 
auf die dänische Mentalität"

schiedliche Funktionen des Phänomens in der interkulturellen Kommunikation werden dabei betont: in deutsch-dänischen Interaktionsphasen sollte der Gebrauch von Humor daher mit äußerster Vorsicht angegangen werden, denn die deutschen und die dänischen Normalitätsvorstellungen dazu, wie man Humor angemessen einsetzt, entsprechen nicht immer den Normen der jeweils anderen Kultur. Vielmehr ist die Wahrscheinlichkeit groß, dass sie als Normzweifelfälle, Normabweichungen oder sogar als Normenkonflikte aufgefasst werden. Die Analysen zu anderen Phänomenen auf der Ebene der Interaktonsphasen zeigen ähnliche Ergebnisse (cf. Müller/Hallsteinsdóttir 2015b): Es sind nicht nur die sprachliche Realisierung und die Inhalte der Kommunikation, sondern genauso oft der Kontext und das Timing, die die Angemessenheit des Handelns in einer konkreten Situation beeinflussen. So zeigen sich z. B. thematisierten Unterschiede in der E-Mailkommunikation sowohl im Wortlaut, Grad der Formalität, Art und Menge der Informationen als auch in den als üblich angesehenen Antwortzeiten.

Die Ebene der Interaktionen wurde oben mit Beispielen 8-11 durch das Phänomen Professionalität und Seriosität illustriert. Hier wird ein in anderen Ratgebern und auch in den SMiKFragebogenerhebungen häufig erwähntes Thema angesprochen: unterschiedliche Handlungsmuster und Erwartungen an bestimmte Handlungsmuster, die in der deutsch-dänischen Wirtschaftskommunikation Missverständnisse verursachen. Die Normalitätsvorstellungen davon, welche Handlungen und Verhalten Professionalität und Seriosität signalisieren bzw. als professionell und seriös wahrgenommen werden, werden in der deutsch-dänischen Interaktion auf die Probe gestellt. Diese unterschiedlichen Vorstellungen tragen, wenn sie nicht kritisch bewertet werden, entscheidend zu der negativen Stereotypisierung von den als typisch dänisch und typisch deutsch empfundenen Eigenschaften und Verhalten bei.

Unterschiedliche deutsche und dänische Normvorstellungen zeigen sich ebenfalls deutlich in den Beispielen 12-16 auf der Ebene der sozialen Situationen. Hier ist die Verortung des Phänomens Dresscode in der eigenen Kultur, und die Bewusstmachung, dass es diese als Lebenswelt gibt, ein Ausgangspunkt für Aussagen darüber, dass das, was als Freizeitkleidung in einer Kultur gilt, in einer anderen Lebenswelt als Arbeitskleidung angemessen sein kann. Umgekehrt gilt auch, dass die in einer Kultur angemessene berufliche Kleidung in einer anderen Kultur als unangemessen oder sogar als ein Zeichen für fehlende Professionalität wahrgenommen wird.

Auf der Organisationsebene des Modells wurde das Phänomen der hierarchischen Strukturen eingeordnet. Dass es unterschiedliche Organisationskulturen und Traditionen gibt, auch innerhalb einer Sprachgemeinschaft, ist wohlbekannt. Die Beispiele 18-23 deuten an, dass die unterschiedliche hierarchische Organisierung ein Aspekt der als auffällig wahrgenommenen deutsch-dänischen Unterschiede ist. Diese Unterschiede prägen das Verhalten und sie tragen auch zu der Erklärung von einem in Relation zur eigenen Normvorstellung als auffällig wahrgenommenen Verhalten bei.

Die übergeordnete, alle anderen Ebenen des verwendeten Modells umfassende ist die Ebene der Kulturen. Auf dieser Ebene haben wir das Phänomen Arbeitskultur eingeordnet. Die Beispiele 24-26 zeigen einen aus deutscher Sicht sehr auffälligen Unterschied im Stellenwert der Arbeit einerseits und von Familie und Freizeit andererseits, der sich in einer sehr positiven 
Auffassung der deutschen Interviewten von dänischer Work-Live-Balance in der Kultur am Arbeitsplatz manifestiert.

Durch die Anwendung des modifizierten Analyseverfahrens der linguistischen Sprachkritik und die Einordnung in das Modell zur situativen Differenzierung des Kontextes konnten die Aussagen aus den Interviews ausgewertet und in einer Form dargestellt werden, die sich auch für die Umsetzung in Ratgebermaterialen als nützlich erwies. Die Interviewzitate wurden in den Ratgebermaterialen durch die Ergebnisse aus andere SMiK-Untersuchungen, und durch Illustrationen von einer professionellen Graphikerin ergänzt. In dieser Weise ausgewertet zeigt sich die Vielfältigkeit der - aus dänischer oder deutscher Sicht - als auffällig wahrgenommenen sprachlich-kulturellen Phänomene: Es geht nicht darum, dass deutsche Geschäftsbeziehungen immer Ordnung haben wollen, oder dass die dänischen Unternehmen alle, lässig' sind, sondern es gibt spezifische Situationen in denen es in Bezug auf unterschiedliche Aspekte und Ebenen der deutsch-dänischen Kommunikation potenzielle Abweichungen von Normen, Konflikte mit Normen oder Normzweifelfälle gibt. Solche Abweichungen können generalisierende Auffassungen verfestigen - und somit den in den SMiK-Untersuchungen festgestellten stereotypen Vorstellungen führen. Das von uns eingesetzte Modell ermöglicht einen differenzierten Zugang, und damit auch eine differenzierte Darstellung der in den Interviews festgestellten interkulturell als auffällig thematisierten Phänomene.

Das Modell hat jedoch auch gewisse Grenzen. Die Ebenen sind in den Daten nicht immer klar abgrenzbar und sie greifen ineinander über. So können die interkulturell auffälligen Phänomene nicht immer eindeutig einer Ebene zugeordnet werden, denn der interkulturellen Kommunikation liegen komplexe kulturelle Strukturen zu Grunde, die mit unserem Modell zwar sinnvoll ausdifferenziert, nicht jedoch komplett in ihrer interkulturellen Komplexität erfasst werden können. Ein Beispiel wurde bereits auf der Ebene der Interaktionen angesprochen. Die Thematisierung von Professionalität und Seriosität wurde ebenfalls auf der Ebene der sozialen Interaktionen eingeordnet, dort aber durch andere Thematisierungen aus den Interviews verankert. Über die Einordnung entscheidet die Art der Thematisierung des Phänomens: Werden darin Aussagen zur konkreten Interaktion zwischen z. B. Geschäftspartner gemacht oder beziehen sich die Thematisierungen eher auf Situationen, in denen es unterschiedliche deutsche und dänische Normvorstellungen gibt?

Ein weiteres Beispiel sind Thematisierungen der Anrede mit Sie oder Du. Das Siezen und Duzen kann durch eine Verortung des Phänomens in der Sprache auf der Ebene der sprachlichen und nicht-sprachlichen Zeichen eingeordnet werden. Dennoch wird das Duzen und Siezen im deutsch-dänischen Kontext oft als ein Teil vom Phänomen Höflichkeit in der Interaktion gesehen. Dadurch, dass das $D u$ die unmarkierte Anrede im Dänischen ist, führt eine unreflektierte Übernahme der jeweils muttersprachlichen Anrede beim Sprachenwechsel in der deutsch-dänischen Kommunikation gelegentlich zu dem Missverständnis, dass es den Dänen an Höflichkeit mangelt, wenn sie auf Deutsch kommunizieren. Dies liegt u. a. daran, dass die dänischen Normen für die angemessene Anrede von Geschäftsverbindungen nicht primär in der Sprache verortet sind, sondern eher auf der Ebene Interaktionsphasen (siehe Abschnitt zu Humor) zuzuordnen sind.

Trotz dieser Grenzen zeigt die in den Analysen vorgenommene Verortung der Textbeispiele auf den Ebenen des Modells, dass die sprachlichen, situativen, individuellen, sozialen und 
auf die dänische Mentalität"

kulturellen Aspekte der komplexen Handlungs- und Kommunikationsstrukturen in der deutsch-dänischen Kommunikation durch unsere Analysen ausdifferenziert und nachvollziehbar gemacht werden können. Die Form des Modells in Abbildung 2 soll daher verdeutlichen, dass alle Ebenen immer in jeder Situation vorhanden sind, diese in einander übergreifen und sich gegenseitig beeinflussen, die darin verorteten Phänomene aber nicht immer gleich stark die Normalitäts- und Normvorstellungen der in der jeweiligen Situation repräsentierten Lebenswelten prägen.

Die hier vorgestellten Beispielanalysen zeigen, dass die Einordnung der in den Interviews als auffällig wahrgenommenen Phänomene in das Modell zur Differenzierung des Kontextes eine Konkretisierung der möglichen interkulturellen Abweichungen von Normen, Konflikte mit Normen oder Normenzweifelfälle in verschiedenen Kontexten der deutsch-dänischen Kommunikation ermöglicht. Dadurch, dass die Aussagen der Interviewteilnehmer nicht als ontologisch geltende Wahrheiten angenommen werden, sondern kritisch und als situativ und kulturell bedingte Thematisierungen von Erfahrungen in konkretisierbaren Situationen in das Modell eingeordnet werden, können für die Kommunikation unbrauchbare Generalisierungen von den Deutschen und den Dänen vermieden werden. Denn die Beschreibung der hier dargestellten Analyseergebnisse zu als auffällig wahrgenommenen Thematisierungen interkultureller Unterschiede ließe sich leicht in generalisierende Charakterisierungen von dänischer und deutscher Kultur und Normen umsetzen. Durch ihre Einordnung in das Modell werden jedoch die in den Interviewdaten festgestellten - von den Interviewten in der Praxis erfahrenen deutsch-dänischen Unterschiede an ihrem Vorkommen in konkreten Situationen verortet und somit für die Kommunizierenden nachvollziehbarer gemacht, als es bei generalisierenden Beschreibungen von stereotypen Vorstellungen oder Verhaltensweisen der Fall sein kann. Die Resultate aus den Interviews werden demnach hier nicht in Generalisierungen darüber, wie die Dänen und die Deutschen sind - damit würden wir die Stereotypisierung der Anderen noch verstärken - zusammengefasst. Stattdessen werden die Analyseergebnisse in Orientierungen implementiert, die ihren Ausgangspunkt in konkreten Kommunikationssituationen haben, und in denen das Bewusstsein der Sprecher für die funktional angemessene Kommunikation in Relation zu den Ebenen unseres Modells trainiert wird. Das Ziel ist die Bewusstmachung der eigenen Kulturbrille und der Aufbau von individueller Mehrkulturalität, um in der interkulturellen Kommunikation funktional angemessen - und somit erfolgreich - handeln zu können.

Die Analyseergebnisse zeigen zudem, dass Stereotype trotz ihres eher schlechten Rufs nicht nur als negative Vorurteile, sondern vielmehr als musterhafte mentale Konzeptionen aufgefasst werden sollten, die als sprach- und kulturbestimmte Denk- und Handlungsmuster sowohl positiv als auch negativ eingesetzt werden können. Die Analyse der Interviewdaten zeigt, dass Stereotypisierungen ein selbstverständlicher Teil der Erklärungen über die Anderen sind, und dass Stereotype auf vielen Ebenen thematisiert werden, auch wenn das Thema Stereotype nicht gezielt in den Interviews angesprochen wurde. Die Interviewten greifen dabei auf die eigene Erfahrung, kulturelles Verständnis und Bewusstsein sowie eventuelles Fachwissen und generalisierende Auffassungen zurück. 


\section{$5 \quad$ Ausblick}

Die Verortung der Ratgebermaterialien in dem Paradigma der linguistischen und didaktischen Sprachkritik mit Fokus auf das Konzept der funktionalen Angemessenheit sowie die Einordnung der interkulturellen Kommunikation in Anlehnung an Boltens Kulturverständnis (cf. Bolten 2007b, 2012, 2014, 2015) hat sich als ein sehr brauchbarer Ansatz erwiesen, um empirisch abgesicherte und theoretisch-methodisch fundierte Materialien für die deutschdänische Kommunikation zu erstellen.

In der Diskussion über Sprach- und Kulturgemeinschaften wird die Container-Metapher oft bemüht. Damit wird die interkulturelle Kommunikation häufig als eine Aushandlung zwischen zwei klar abgegrenzten Kulturen aufgefasst. Eine Konzeptualisierung von (nationalen) Kulturen als abgeschlossenen Einheiten stellt allerdings ein praktisches Problem bei der Vermittlung der Ergebnisse aus den SMiK-Untersuchungen dar, denn die interkulturelle Kommunikation findet immer zwischen Individuen in konkreten Situationen statt. Hier stellt sich die Frage danach, wie Thematisierungen von dänischen und deutschen Handlungsmustern, die in den Interviews teilweise als stark stereotype, generalisierende Vorstellungen auftreten, sowie die darauf basierenden Empfehlungen und Handlungsanweisungen, so dargestellt werden können, dass sie nicht zu einer Verfestigung von Stereotypen und somit einer Verfremdung der anderen Kultur führen. Vielmehr sollen die Kommunizierenden - im Sinne der didaktischen Sprachkritik - in die Lage versetzt werden, dass sie die sprachlichen und nichtsprachlichen Mittel angemessen einsetzen, so dass sie ihre Ziele in der deutsch-dänischen Kommunikation erreichen können. Entscheidend ist dabei nicht der Aufbau einer speziellen interkulturellen Kompetenz, sondern, wie im Abschnitt 2.4 dargestellt wurde, die Entwicklung von eigenkulturellen Schlüsselkompetenzen wie Empathie, Rollendistanz und metakommunikativen Fähigkeiten, die für erfolgreiches Handeln grundlegend sind.

Mit der Auffassung von einer situativ verankerten funktionalen Angemessenheit in der Kommunikation und dem im Abschnitt 2.3 eingeführten Konzept von Mehrkulturalität wird die Vorstellung von interkultureller Kommunikation zwischen zwei getrennten Kulturen aufgebrochen. Eine wichtige Grundlage hierbei ist eine offene, soziale fixierte Kulturauffassung (cf. Abschnitt 2.1), die eine Zugehörigkeit zu mehreren Lebenswelten (cf. Bolten 2007b, 2012) und somit eine (mentale) Mehrkulturalität zulässt. Die Idee der Kulturbrille wird in der individuellen Lebenswelt der jeweiligen Leserinnen und Leser verankert. Diese Lebenswelt wird durch Stereotype, d. h. sprachliche und kulturelle Denk- und Handlungsmuster und Vorstellungen von Normen und Normalität, strukturiert. Die Kulturbrille verweist jedoch auf die Gebundenheit dieser Muster und Normen an Situationen, Strukturen und die Erfahrungen und Ideale in der Lebenswelt der Leserinnen und Leser selbst. So übernehmen wir auch hierin die Auffassung der linguistischen Sprachkritik (cf. Abschnitt 3.1): Wir setzen keine Normen, sondern wir machen Normalitäts- und Normvorstellungen durch Analyse und Beschreibung nachvollziehbar, um eine Orientierung für die deutsch-dänische Kommunikation zu geben.

In den Ratgebern haben wir die zentrale Rolle von eigenen und fremden Normen und Normalitätsvorstellungen zu Sprachen und Kulturen in der Kommunikation hervorgehoben. Wir argumentieren dafür, dass der Begriff der Angemessenheit als die funktional angemessene Einhaltung von Normen und Erwartungshaltungen nicht nur für die Analyse von sprachlichen Phänomenen (cf. Kilian/Niehr/Schiewe 2010: 41, cf. Abschnitt 3.2) anwendbar ist. Vielmehr 
auf die dänische Mentalität"

kann durch das Konzept der funktionalen Angemessenheit ein Zugang aufgezeigt werden, mit dem komplexe interkulturelle Phänomene auf mehreren Ebenen der Kommunikationssituation erfasst und eingeordnet werden können. Mit diesem Konzept, das haben wir in diesem Beitrag gezeigt, können auch Ergebnisse aus Analysen von empirischen Daten eingeordnet werden, die den kritisch-bewussten Umgang mit sprachlich-kulturellen Phänomenen, Handlungsmustern und stereotypen Vorstellungen in der interkulturellen Kommunikation fördern.

Das übergeordnete Ziel einer sprachkritischen Einordnung, wie wir es mit den dargestellten Ratgebermaterialien realisiert haben, ist es, „sprachliches [und non-verbales] Handeln in Richtung auf ,gelingende Kommunikation“ zu befördern.“ (Kilian/Niehr/Schiewe 2010: 52). In der interkulturellen Kommunikation geht es zudem darum, die Kommunizierenden dazu zu befähigen, nicht nur das „Anderssagen“ (Kilian/Niehr/Schiewe 2013: 311) sondern auch und insbesondere - das Anderssein wahrzunehmen. Eine solche Wahrnehmung ist die Voraussetzung dafür, dass nicht nur die eigenkulturellen Normen, sondern auch die Normen und Normalitätsvorstellungen der anderen Kultur erkannt, respektiert und im Sprach- und Kulturgebrauch der interkulturellen Kommunikation angemessen angewendet werden können.

In den Ratgebern fokussieren wir auf die Unterschiede und Ähnlichkeiten in sprachlichkulturellen Normen, die konkrete Kommunikationssituationen beeinflussen können. Die Ratgeber sind darauf ausgelegt, das Wissen der Benutzerinnen und Benutzer über deutschdänische Unterschiede und Ähnlichkeiten zu vertiefen sowie ein Bewusstsein für funktional angemessenes Handeln in der deutsch-dänischen Kommunikation aufzubauen. In Anlehnung an Kilian/Niehr/Schiewe (2013: 311), die ,die Befähigung zur Wahrnehmung des ,Anderssagens““ als „eine der wichtigsten Aufgaben der didaktischen Sprachkritik und des Deutschunterrichts“" ansehen, betrachten wir daher die Befähigung zur Wahrnehmung und Reflexion über das Anderssein und das Sich-Anders-Verhalten als weitere wesentlichen Voraussetzungen für die gelungene interkulturelle Kommunikation. Die Förderung dieser Befähigung sehen wir somit als eine der wichtigsten Aufgaben eines (sprach)kritischen Mutter- und Fremdsprachenunterrichts, in dem die Grundlagen für eine gelungene interkulturelle Kommunikation vermittelt werden sollten.

\section{Literatur}

Ahrendt, Birte/Kiesendahl, Jana (2013): „Funktionale Angemessenheit. Gesprächs- und lehrwerksanalytische Perspektiven“. Mitteilungen des Deutschen Germanistenverbandes 60/4: 336-355.

Bolten, Jürgen (2007a): Einführung in die Interkulturelle Wirtschaftskommunikation. Göttingen: Vandenhoeck \& Ruprecht.

Bolten, Jürgen (2007b): Interkulturelle Kompetenz. 4. vollständig überarbeitete Auflage. Erfurt: Landeszentrale für politische Bildung Thüringen. www.dbthueringen.de/servlets/DerivateServlet/Derivate-20394/interkulturellekompetenz.pdf [04.11.2015].

Bolten, Jürgen (2012): Interkulturelle Kompetenz. 5. ergänzte und aktualisierte Auflage. Erfurt: Landeszentrale für politische Bildung Thüringen. 
www.ikkompetenz.thueringen.de/downloads/1210Bolten_Ik_Kompetenz_Vorversion_5Au fl.pdf [22.05.2016].

Bolten, Jürgen (2014): „Reziprozität, Relationalität und Mehrwertigkeit. Ein Plädoyer für einen holistischen Kulturbegriff“. In: Eidukeviciene, Ruta/Johänning-Radziene, Antje (eds.): Interkulturelle Aspekte der deutsch-litauischen Wirtschaftskommunikation. München, Iudicium: 18-39.

Bolten, Jürgen (2015): Einführung in die Interkulturelle Wirtschaftskommunikation. 2. Auflage. Göttingen: Vandenhoeck \& Ruprecht.

ELAN-Studie (2006): Effects on the European Economy of Shortages of Foreign Language Skills in Enterprise. European Commission.

KMU-Studie (2003): Entwicklung von Kompetenzen und Qualifikationen in KMU. Beobachtungsnetz der europäischen KMU. Europäische Kommission.

Fix, Ulla (2006): „Was heißt Texte kulturell verstehen? Ein- und Zuordnungsprozesse beim Verstehen von Texten als kulturellen Entitäten“. In: Blühdorn, Hardarik/Breindl, Eva (eds.): Text-Verstehen. Grammatik und darüber hinaus. Berlin, de Gruyter: 254-276.

Hallsteinsdóttir, Erla (2013): „Möglichkeiten textbezogener Sprachdidaktik“. Deutsch als Fremdsprache: $93-101$.

Hallsteinsdóttir, Erla/Farø, Ken (2010): „Interlinguale Phraseologie: Theorie, Praxis und Perspektiven/Interlingual Phraseology: Theory, Practice and Perspectives“. Yearbook of Phraseology: 125-158.

Hallsteinsdóttir, Erla/Kilian, Jörg (eds.) (2015): SMiK-Posterpräsentation von der Konferenz $\{$ DEUTSCH $\}$ und $\{$ D̈̈NISCH $\}$ im Stereotyp: Stereotypenwelten und ihre sprachlichkulturellen Konstituierungsformen, 25.-27. Februar 2015 an der Süddänischen Universität in Odense \| SMiK-Plakate fra konferencen \{DANSK\} og $\{$ TYSK\} $i$ stereotyper: stereotypeuniverser og deres sproglig-kulturelle konstitueringsformer, 25.-27. februar 2015 ved Syddansk Universitet $i$ Odense. Odense: SMiK-Projekt. www.stereotypenprojekt.eu/projektresultate-r-1/smik-publikationer-n/smik-publikationen$\mathrm{r} /[04.10 .2015]$.

Hallsteinsdóttir, Erla et al. (2015): Typisch deutsch - typisch dänisch? SMiKUnterrichtsmaterialien zur Bewusstmachung von nationalen Stereotypen. Odense: SMiKProjekt. www.stereotypenprojekt.eu/projektresultate-r-1/smik-unterrichtsmaterialien-zustereotypen-smik-undervisningsmaterialer-om-stereotyper/ [02.11.2015].

Kilian, Jörg (2001): „Kritische Semantik. Für eine wissenschaftliche Sprachkritik im Spannungsfeld von Sprachtheorie, Sprachnorm, Sprachpraxis“. Zeitschrift für Germanistische Linguistik 29: 293-318.

Kilian, Jörg (2003): „Wörter im Zweifel. Ansätze einer linguistisch begründeten kritischen Semantik“. Linguistik online 16/4: 159-170. https://bop.unibe.ch/linguistikonline/article/view/800/1380 [04.10.2015].

Kilian, Jörg (2005): „Assoziative Stereotype. Sprachtheoretische, sprachkritische und sprachdidaktische Überlegungen zum lexikalisch verknüpften Mythos, Aberglauben, Vorurteil“". In: Busse, Dietrich/Niehr, Thomas/Wengeler, Martin (eds.): Brisante Semantik. Neuere Konzepte und Forschungsergebnisse einer kulturwissenschaftlichen Linguistik. Tübingen, Niemeyer: 117-132. 
auf die dänische Mentalität“"

Kilian, Jörg (2008): „Verborgene Weltansichten entdecken. Zur Konzeption einer kritischen Semantik assoziativ-semantischer Stereotype aus sprachwissenschaftlicher, sprachphilosophischer und sprachdidaktischer Sicht. Zugleich eine linguistisch begründete Kritik politisch korrekter Sprachkritik“. In: Pappert, Steffen/Schröter, Melani/Fix, Ulla (eds.): Verschlüsseln, Verbergen, Verdecken in öffentlicher und institutioneller Kommunikation. Berlin, Schmidt: 49-68.

Kilian, Jörg (2015): „Von blonden Däninnen aus deutscher Sicht. Nationale Stereotype und didaktische Sprachkritik - ein Zwischenbericht aus einem laufenden Forschungsprojekt“". In: Peschel, Corinna/Runschke, Kerstin (eds.): Sprachvariation und Sprachreflexion in interkulturellen Kontexten. Frankfurt a. M. etc., Lang: 155-182.

Kilian, Jörg/Niehr, Thomas/Schiewe, Jürgen (2010): Sprachkritik. Ansätze und Methoden der kritischen Sprachbetrachtung. Berlin/New York: de Gruyter.

Kilian, Jörg/Niehr, Thomas/Schiewe, Jürgen (2013a): „Zur Einführung“. Mitteilungen des Deutschen Germanistenverbandes 4: 397-299.

Kilian, Jörg/Niehr, Thomas/Schiewe, Jürgen (2013b): „Es gibt kein Falsches im Angemessenen. Überlegungen zu einem sprachkritischen Analysemodell“". Mitteilungen des Deutschen Germanistenverbandes 4: 300-320.

Kvale, Steinvar (1997): InterView: en introduktion til det kvalitative forskningsinterview. Kopenhagen: Reitzels.

Lippmann, Walter (1922): Public Opinion. New York: Macmillan.

List, Pia/Wagner, Johannes (1992): „Nationale Stereotype im internationalen beruflichen Alltag: Überlegungen anhand eines Fallbeispiels“. In: Grindsted, Annette/Wagner, Johannes (eds.). Communication for Specific Purposes/Fachsprachliche Kommunikation. Tübingen, Narr: 210-226.

Müller, Katarina Le (2016): „Thematisierungen von Sprache in der deutsch-dänischen interkulturellen Kommunikation“. In: Hallsteinsdóttir, Erla et al. (eds.): Perspektiven der Stereotypenforschung. Frankfurt a. M. etc.: Lang.

Müller, Katarina Le/Hallsteinsdóttir, Erla (2015a): Dansk-tysk kulturbrille: SMiK-tjekliste til den interkulturelle kommunikation. Odense: SMiK-Projekt. www.stereotypenprojekt.eu/projektresultate-r-1/interkulturelle-kommunikationinterkulturel-kommunikation/ [28.02.2016].

Müller, Katarina Le/Hallsteinsdóttir, Erla (2015b): Deutsch-dänische Kulturbrille: SMiKLeitfaden zum funktional angemessenen Handeln in der interkulturellen Kommunikation. Odense: SMiK-Projekt. www.stereotypenprojekt.eu/projektresultate-r-1/interkulturellekommunikation-interkulturel-kommunikation/ [28.02.2016].

Müller, Katarina Le/Hallsteinsdóttir, Erla (2015c): Kommunikation med Tyskland - Tips og tricks til succes. Odense: SMiK-Projekt. www.stereotypenprojekt.eu/projektresultate-r1/interkulturelle-kommunikation-interkulturel-kommunikation/ [28.02.2016].

Müller, Katarina Le/Hallsteinsdóttir, Erla (2015d): Kommunikation mit Dänemark - Tipps und Tricks für mehr Erfolg. Odense: SMiK-Projekt. www.stereotypenprojekt.eu/projektresultate-r-1/interkulturelle-kommunikationinterkulturel-kommunikation/ [28.02.2016].

Nordhaug, Odd/Grønhaug, Kjell (1994): "Competences as resources in firms". The International Journal of Human Resource Management 5/1: 89-106. 
Rehrmann-Jørgensen, Doris/von der Banck, Marie Luise (2011): Deutsch-dänische Geschäftskultur - ein Praxisorientierter Zugang. Kopenhagen: REBA.

PIMLICO-Projektbericht (2011): Report on Language Management Strategies and Best Practice in European SMEs: The PIMLICO Project. European Commission.

Verstraete-Hansen, Lisbeth (2008): Hvad skal vi med sprog? Holdninger til fremmedsprog $i$ danske virksomheder $i$ et uddannelsespolitisk perspektiv. Kopenhagen: Copenhagen Business School. 